Contract No. W-7405-eng-26

CHEMICAL TECI'NOLCCY DIVISION

\section{A STUDY OF DROPLET FORMATION FOR PREPARATICN OF SPHERES BY INTERNAL GELATION}

Chihang R. Che

John C. Card

Consilitants

P.A. Haas, A.D. Kyon, and W.D. Bond

Date Published: January 1982

Oak Ridge Station

School of Chemical Engineering Practice

Massachusetts Institute of Technology

C.H. Byers, Director

Oak Ridge National Laboratory

Oak Ridge, Tennessee 37830

Operated by

Union Carbide Corporation

for the

Department of Energy

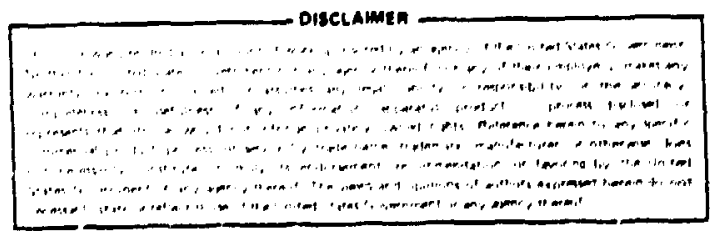


1. Summary $\ldots \ldots \ldots \ldots \ldots \ldots \ldots \ldots \ldots \ldots \ldots \ldots \ldots \ldots \ldots \ldots \ldots, 1$

2. Introduc iun $\ldots \ldots \ldots \ldots \ldots \ldots \ldots \ldots \ldots \ldots \ldots \ldots \ldots \ldots \ldots \ldots, 2$

2.1 Treatment of Nuclear Hastes $\ldots \ldots \ldots \ldots \ldots \ldots \ldots \ldots \ldots . .2$

2.2 Literature Review ............................... 2

2.3 objectives .................................... 4

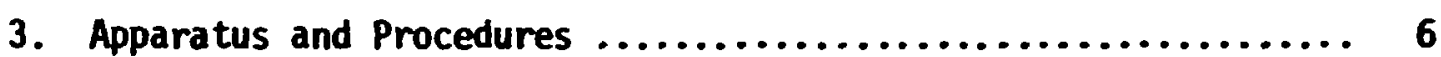

3.1 Apparatus .................................... 6

3.1.1 Droplet-Formation Apparatus ................. 6

3.1 .2 Catcher-Fluid Apparatus ................... 9

3.2 Procedure $\ldots \ldots \ldots \ldots \ldots \ldots \ldots \ldots \ldots \ldots \ldots \ldots \ldots \ldots \ldots \ldots, 9$

3.2.1 Droplet Formation ....................... 9

3.2 .2 Catcher-Fluid Stream ....................... 12

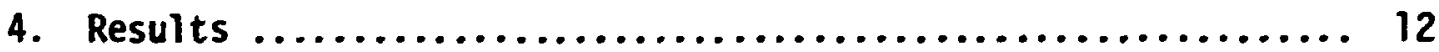

4.1 Experimental observations $\ldots \ldots \ldots \ldots \ldots \ldots \ldots \ldots \ldots \ldots, 12$

4.2 Dimensional Analysis ........................... 18

4.3 Computer Models ................................ 22

4.4 Catcher-Fluid Stream ............................ 22

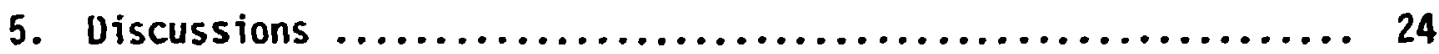

5.1 Limitations of the Correlations .................... 24

5.2 Comparison with the Literature .................... 30

6. Conclusions ..................................... 30

7. Recommendations .................................. 30

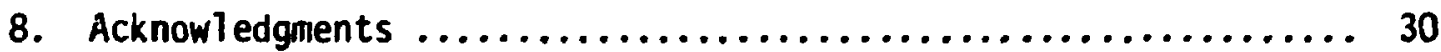

9. Appendix .......................................... 31

9.1 Newtonian-Jet Breakup ........................... 31

9.2 Accurate Calibration Curves ....................... 34

9.3 Dimensional Analysis .......................... 36

9.4 SAS Program Listing and Data Base $\ldots \ldots \ldots \ldots \ldots \ldots \ldots, 38$

9.5 Sample Calculations ............................ 45

9.6 Location of Original Data ....................... 46

9.7 Nomenclature ................................. 46

9.8 Literature References $\ldots \ldots \ldots \ldots \ldots \ldots \ldots \ldots \ldots \ldots \ldots, 48$ 


\section{SURARY}

Internal gelation has been suggested as a means of permanently immobilizing highly radioactive nuclear wastes from the Savannah River Plant of the DOE. These wastes would be incorporated ints a gel structure in the form of small, hardened ceramic spheres, and as such, could be easi?y transported and safely stored. To form these spheres by internal gelation, a chilled feed broth containing the wastes, matrix material, and other components is fed to a vibrating nozzle, forming a jet wich breaks up into a stream of droplets in the air. These droplets then fall into a hot forming liquid, where the release of ammonia triggers a gelation reaction. The spheres are then coated for additional protecticn against leaching. To ensure a uniform coating, the spheres must be of uniform size.

The critical step in forming spheres of uniform size is good droplet formation at the vibrating nozzle. An experimental study was conducted to investigate the factors affecting droplet formation. Three different fiuids were investigated, whose physical properties cover the range which will be encountered in the feed broth. Three stainless-sttol nozzles and one glass unit in the diameter range 325-586 $\mu \mathrm{m}$ were used with jet velocities varying from 1 to $10 \mathrm{~m} / \mathrm{s}$, which allowed the generation of an extensive data base. The important dimensionless groups, such as Reynolds (Re), Heber (We), Froude (Fr), and Power ( $\mathrm{Po}$ ) numbers, and a dimensionless droplet spacing $\left(\lambda / D_{n}\right)$ were identified by a dimensional analysis. The data were then correlated as a power function of these groups. Two correlations resulted. The upper bound of good droplet formation, which corresponded to the maximum allowable flow rate at a fixed frequency, was given by:

$$
\frac{\lambda}{D_{n}}=1.66 \operatorname{Re}^{-0.00043} \mathrm{We}^{-0.43} \mathrm{Fr}^{0.38} \mathrm{Po}^{-0.037}
$$

The lower bound, representing the minimum flow rate when the frequency was fixed, was given by:

$$
\frac{\lambda}{C_{n}}=7.32 \mathrm{Re}^{0.016} \mathrm{We}^{-0.066} \mathrm{Fr}^{-0.049} \mathrm{Po}^{-0.020}
$$

A further obstacle to good gelled-sphere formation is the r.oalescence of droplets at the surface of the hot forming liquid at high vibrator frequencies and flow rates. The use of a catcher stream, which traps the droplets in the air and transports them bclow the surface of the forming 1 iquid, was investigated. The catcher fluid, trichloroethylene (TCE), was found to remain as a liquid sheet when it was directed onto a glass plate. It was recommended that this catcher-stream design and the two correlations be applied at the pilot plant at ORNL. 


\section{IRTRODUCTION}

\subsection{Treatment of Muclear Mastes}

Over the past twenty-five years, highiy radioactive nuclear wastes have accumulated at the Savannah River Plant (SRP) of the Department of Energy. These wastes are derived frow the chemicai reprocessing of nuclear reactor fuel and target elemenis from production reactors. They are alkaline slurries of primarily hydrous oxides of iron, aluminum, and manganese that have been precipitated with excess $\mathrm{NaOH}$. Presently. these wastes are stored in high-integrity, double-walled tanks at SRP. Several methods for the permanent immobilization of these high-level wastes are presently being developed as part of the Department of Energy's plan for long-term management of all federally omed reprocessing waste $(3, \underline{15})$.

A proposed inmobilization method is incorporation of the SRP sludge solids into a gel structure in the form of sma?l spheres (approximately 1-mim diameter). Once they are washed, calcined, and coated, these hardened, ceramic spheres offer excellent resistance to leaching and are easily transported and stored.

Internal gelation [using a feed bro:h containing the waste, matrix components, urea, and hexamethylenetetramine (HIA) that is chilled, mixed, and fed to a vibrating nozzle] is the proposed sphere-production method. The jet, which leaves the nozzle, breaks up into a streali: of droplets in the air. Subsequently, the droplets enter a hot organic liquid, such as trichloroethylene (TCE) or silicone oil, where the HWTA decomposes, releasing ammonia, which causes gelation of the droplets. The gelled spheres are then washed, dried, and calcined. For additional protection against leaching, a coating of pyrolytic carbon is applied to the spheres. Finally, for increased mechanical strength and protection against oxidation, the spheres are coated with $\alpha-\mathrm{Al}_{2} \mathrm{O}_{3}$.

To ensure that a uniform coating is applied to tive spheres in the coating process, spheres of a uniform size are desired. Since the spheres are a direct result of the droplets formed by the vibrating nozzle, the critical step in the uniform-sphele size is the formation of uniform droplets by the vibrating nozzle. The following section discusses a method for obtaining uniform droplet.

\subsection{Literature Review}

According to the classical mechanism postulated by Lord Rayleigh, a free coluinn of 1 iquid is unstable if its length is greater than its circumference (14). The process by which drops are produced from a liquid stream oepenos on tne nature of the flow such as the way in which energy is imparted to the 1 iquid, the properties of the liquid (viscosity, density, and surface tension), and the properties of the ambient atmosphere (2). 
One practicai way to produce droplets is through the use of a pulsed nozzle. If a jet is excited by a small-amplitude axial vibration of appropriate frequency, the jet breaks up into uniformly spaced drops. The size of the drops can be controlled by the liquid flow rate and by the frequency of vibration imposed on the jet. It is assumed that one drop is formed per cycle of vibration per orifice (16). Therefore, a simple mass balance gives:

$$
Q=\left(\frac{\pi D^{3}}{6}\right)+N
$$

where:

$$
\begin{aligned}
& Q=\text { volumetric flow rate of liquid } \\
& \mathbf{f}=\text { frequency } \\
& \boldsymbol{N}=\text { number of orifices }
\end{aligned}
$$

For a single nozzle, Eq. (1) reduces to the following expression for droplet diameter:

$$
D=\left(\frac{6}{\pi} \frac{Q}{6}\right)^{1 / 3}
$$

For the jet to $k$ stable, however, the droplet diameter can only fall within a certain range. Merrington and Richardson (12) found that with nozzles up to $1000 \mathrm{Lm}$, the drop size obtained at low velocities was roughly twice the nozzle diameter. The ratio of drop diameter to nozzle diameter ranged from 1.8 to 2.3 .

Aithough a pulsed nozzle is good for procucing uniform drops, it also has a tendency to form relatively smaller droplets interspersed among the main drops (satellite drops), which is undesirable in the internal gelation process. Several satellite studies focused on the region of operating conditions, which would suppress the formation of satellites.

In a paper by Pimbley anci Lee (13), a second-order spatial-instability analysis was used to simulate the formation of satellites from the main drops. According to Pimbley and Lee, the two most important parameters which control satellite formation are the amplitudp of the perturbation and the wavelength-to-diameter ratio. A satellite drop can separate from a larger drop on the fore side of the satellite first, on the aft side first, or on both ends simultaneously. The time interval between the two separat?ons is defined as the satellite interaction time. Within this time period momentum transfer occurs, which r'ssults in the slowing down or speeding up of the satellite relative to the main stream. With very small perturbation amplitude, the first separation occurs on the fore 
side of the satellite, producing a slightly lower satellite velocity. At a later time, the satellites will merge with the main drops, causing nonuniform droplet sizes. Visuaily, the jet thus contains rear-merging satellites. Figure IA schematically illustrates such a situation.

When the perturbation amplitude is increased, a point is reached where the two ends of the satellite break off simultaneously. No momentum transfer takes flace, and the satellites move with the same veiocity as the main stream. This is the infinite satellite condition show in Fig. IB. When the amplitude is increased beyond this point, the jet breaks up at the aft side of the satellites first, producing forward-merging satellites as shown in Fig. IC.

In sumary, a pulsed nozzle can produce uniform spherical droplets, although severai parameters seem to affect the uniformity of the droplets. A major difficulty is the formation of satellite droplets. At Oak Ridge National Laboratory (ORNL), previous work applied this technique to the internal gelation process $(7,8, \underline{9}, 18)$. However, the cperating conditions for good droplet formation were mostly determined by trial-anderror. A study was needed to define the limits of operating range.

Once formed in the air, the droplets must enter the heated organic fluid without coalescence. The researchers at ORNL found that at high frequency of droplet formation and high velocities, the droplets tended to coalesce as they struck the surface of the heated organic medium, rather than passing into it and hardening into uniform-size spheres. To transport the droplet into inis forming liquid and to avoid this coalescence at the surface, a catcher stream can be used. Droplets formed at the nozzle fall into a passing stream, which conveys them below the surface of the forming liquid. While some catcher-stream concepts have been suggested, an extension of these ideas is necessary before they will be usable, particularly with a TCE medium. Later a study of the hydrodynamics of sich a configuration will be necessary.

\subsection{Objectives}

The objectives of this study were:

1. To study the effect of viscosity, density, and surface tension of the fluid, oscillation frequency and power input to the nozzle, orifice diameter, and fluid flow rate upon the range of good droplet formation.

2. To develop a correlation to describe the operable range of all such variables.

3. To investigato the use of a catcher-fluid stream, which traps and carries the droplets into the organic medium, especia?ly at high frequencies. 


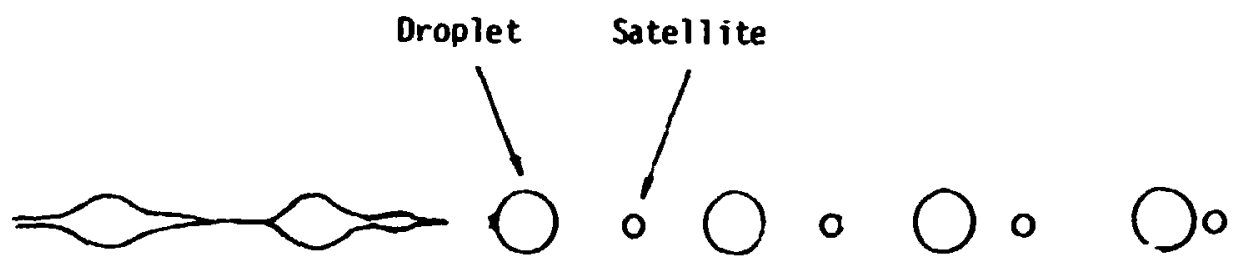

A) Rear-Herging Satellites

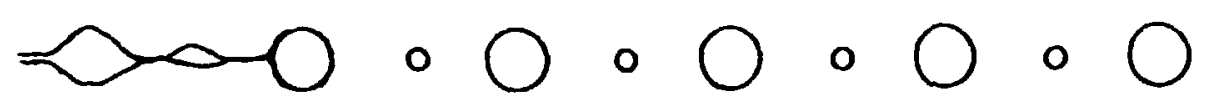

B) Infinite Satellite Condition

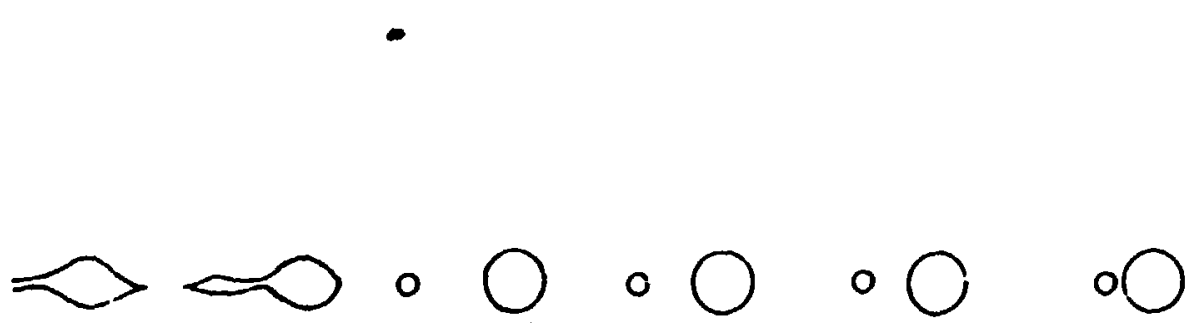

C) Forward-Merging Satellites

MASSACHUSETTS INSTITUTE OF TECHWOLOGY SCHOOL OF CHEMICAL ENGINEERING PRACTICE oAK RIDGE NATIÓNAL LaP JRatoRY

THREE TYPES OF SATELLITE FORMATION 


\section{APPARATUS AND PROCEDURE}

\subsection{Apparatus}

\subsubsection{Droplet-Formation Apparatus}

The central feature of the experimental apparatus (see Fig. 2) was a steel or glass nozzle with a circular orifice, which was attached to the diaphragm of the electromechanical vibrator (Aipha-H Corp. Model AV-6), sc that it produced axial vibration of the fluid stream. The shaft of the nozzle was mounted at a $45^{\circ}$ angle below horizontal. Four stainless steel nozzles and one glass nozzle were used in the expe iments (see Fig. 3 ). The 180-un orifice, however, was apparently too narrow, and it was not possible to issue a liquid jet from any of the test liquids.

The orifice of each nozzle was examined under a microscope (50X magnification); the orifices of the stainless-steel nozzles were circular at least to the extent that their shape was visible, while the orifice of the glass nozzle was somewhat eliptical (maximum diameter/minimum diameter = 1.2). The edges of all orifices appeared very rough. Other nozzle characteristics are listed in Table 1.

Table 1. Nozzle Characteristics

\begin{tabular}{llccc}
\hline $\begin{array}{l}\text { Orifice } \\
\text { Diameter } \\
(\mathrm{mm})\end{array}$ & $\begin{array}{c}\text { Material of } \\
\text { Construction }\end{array}$ & $\begin{array}{c}\text { Weight } \\
(\mathrm{c})\end{array}$ & $\begin{array}{c}\text { Straight Tubing Section } \\
\text { Before Orifice } \\
(\mathrm{cm})\end{array}$ \\
\cline { 2 - 3 } 180 & stainless steel & 13 & 1.3 \\
325 & stainless steel & 13.78 & 1.3 \\
350 & stainless steel & 13.92 & 1.3 \\
501 & stainless steel & 12.62 & 1.3 \\
586 & glass & 10.60 & 3.8 \\
\hline
\end{tabular}

The vibrator was driven by a 0-to-25-watt power amplifier (1) (A)pha-M Corp. Model $\mathrm{OC}-25$ ! with a frequency range of 20 to $20,000 \mathrm{~Hz}$. An AC ammeter on the front panel of the amplifier and a Simpson Model 313 voltmeter permitted the vibration power to be calculated. The frequency of the vibrator-drive signal was accurately measured $( \pm 0.1 \mathrm{~Hz})$ with a digital frequency counter (HP Model 5308A), while a stroboscope (General Radio Model 1538A Strobotac) allowed the droplets to be observed. The stroboscope frequency was set at a fractional harmonic (usually within 


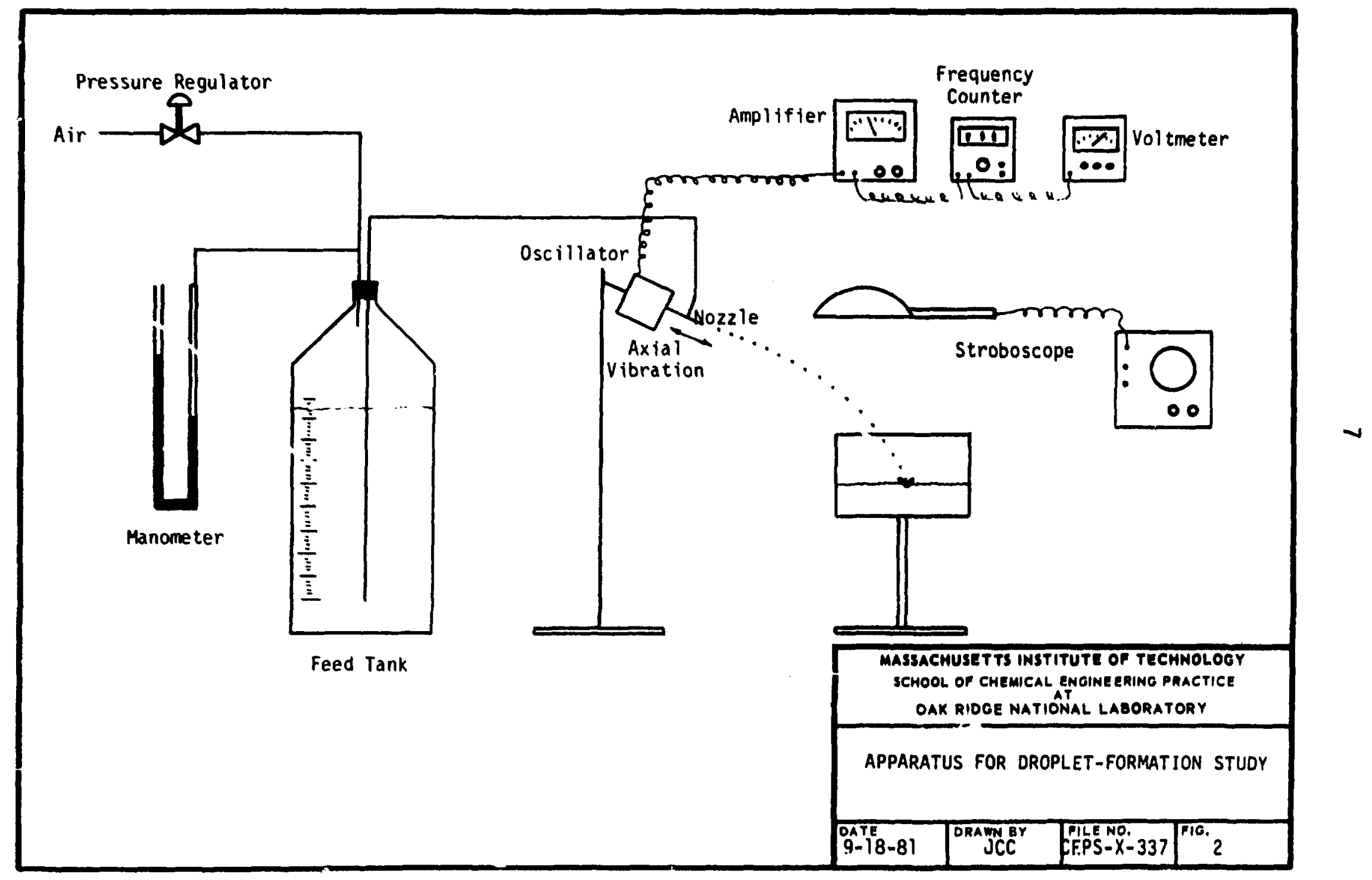




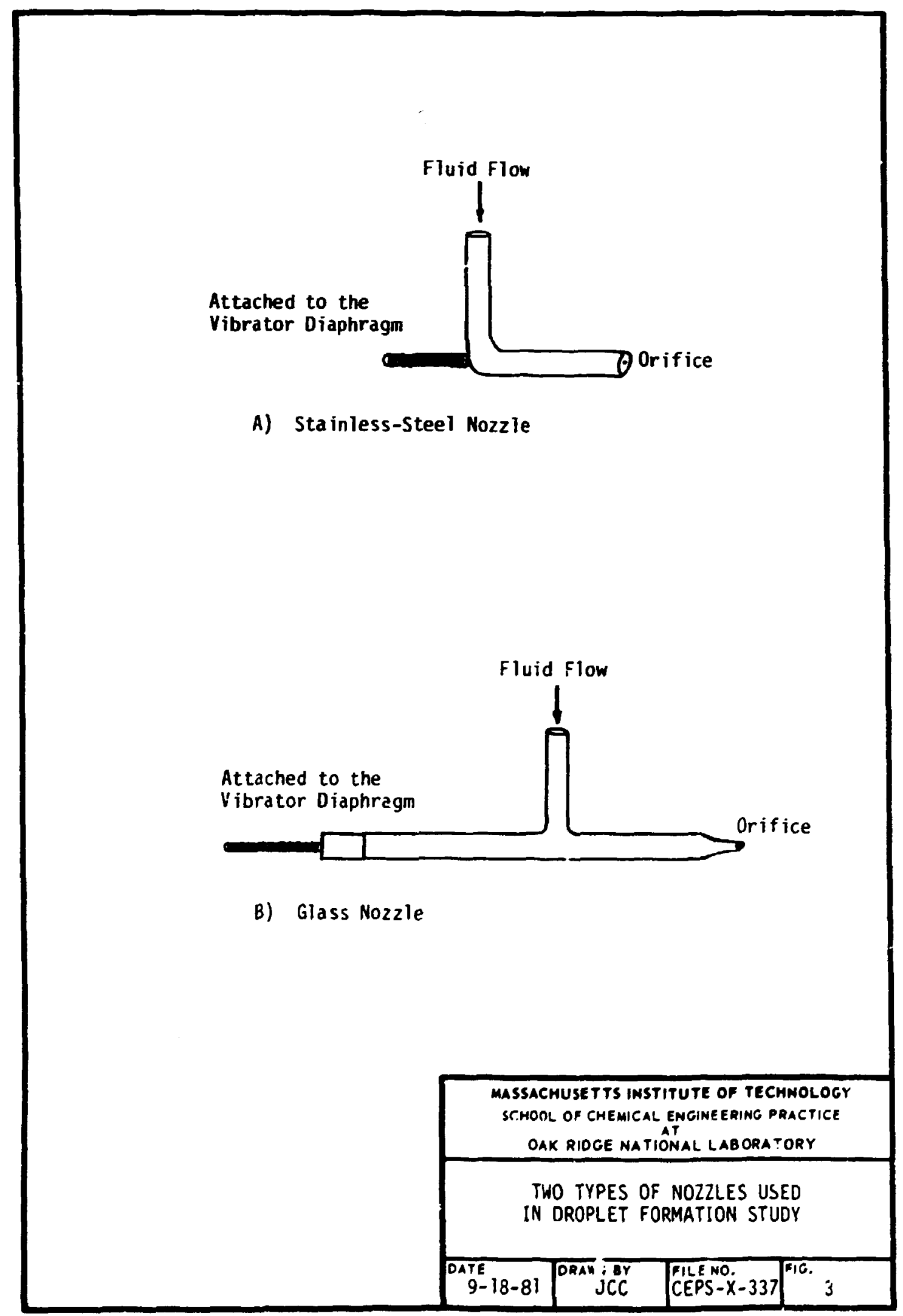


the range of $1 / 10$ to $1 / 50$ ) of the vibrator frequency. This made the droplet stream appear stationary and facilitated measurements of droplet spacing.

The test liquid flowed to the nozzle from a pressurized feed tank through flexible tubing to avoid the introduction of extraneous signals to the nozzle. Fluid flow rate was controlled by regulating the air pressure above the liquid (0-50 $\mathrm{cm}-\mathrm{Hg})$; this pressure was measured on a mercury manometer. The liquid level in the feed tank was measured with a ruler, so that its static head contribution to the total pressure upstrean of the nozzle could be calculated. The static head ranged from 0.7 to $38.3 \%$ of the total pressure. However, its contribution to the total pressure changed at most by $1.5 \%$ during any single run.

\subsubsection{Catcher-Fiuld Apparatus}

Figure $4 A$ is a schematic diagram of the catcher-fluid stream apparatus. A close-up side view of the catcher-stream nozzle is shown in Fig. 4B. The nozzle was constructed from a 10-in. section of $\frac{1}{2}-$ in.- 00 stainlessstee t tubing, which was sealed at one end. A slot $2.54 \mathrm{~cm}$ long and $500 \mu \mathrm{m}$ wide was cut into the wall of the tubing, $2.5 \mathrm{~cm}$ from the ent. A needle valve was attached to the other end to control the catcher-fiuid flow rate out of the slot.

The catcher fluid trichloroethylene (TCE) flowed from a pressurized feed tank similar to the one used for the test liquid. The catcher fluid issued from the slot as a flat sheet $2.5-\mathrm{cm}$ wide and approximately $0.5-\mathrm{mm}$ thick. The thickness of the jet was very non-uniform and many ripples were present, making the thickness vary from about 0.3 to $0.7 \mathrm{~mm}$ a long the width of the jet. At approximately $2.5 \mathrm{~cm}$ from the nozzle, the jet collapsed into a single thread.

To provide a catcher stream with a more uniform surface, the catcher fluid was directed onto a $15-\mathrm{cm}$ square sheet of Pyrex glass plate. The nozzle was mounted approximately $1 \mathrm{~cm}$ above the surface of the glass. The catcher fluid then flowed out of the slot and onto the glass surface, expanding to an 8-cm-wide film with $0.2 \mathrm{~mm}$ thickness as it flowed down the plat.a. The surface of the catcher-fluid film on the glass was much more uniform than that of the free jet. The droplet stream was directed into this moving film.

\subsection{Prccedure}

\subsubsection{Droplet Formation}

Three fluids were used in this study. Their physical properties of interest are listed in Tahle 2. 


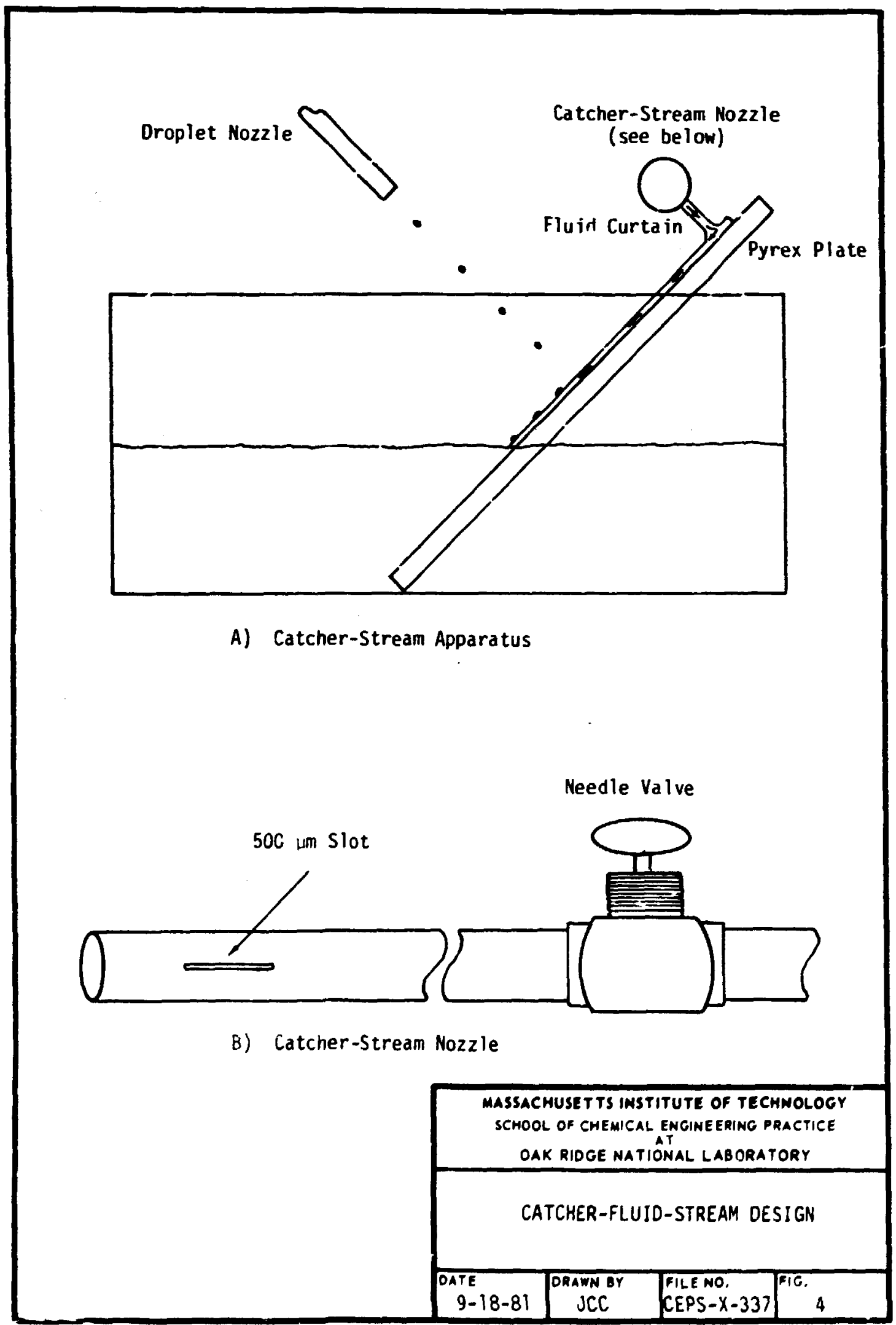


Tatie 2. Physical Properties of Model Fluids at $20^{\circ} \mathrm{C}$

\begin{tabular}{|c|c|c|c|}
\hline Fluid & $\begin{array}{c}\text { Viscosity } \\
(\mathrm{Pa}-j) \\
\end{array}$ & $\begin{array}{l}\text { Density } \\
(\mathrm{kg}-\mathrm{m}-3)\end{array}$ & $\begin{array}{l}\text { Surface Tension } \\
\left(\mathbb{N}-m^{-1}\right)\end{array}$ \\
\hline Etnylene glycol & $1.99 \times 10^{-2}$ & 31113 & $4.77 \times 10^{-2}$ \\
\hline $20 \%$ glyceral (aqueous) & $1.769 \times 10^{-3}$ & 1046.9 & $7.2^{\prime} \times 10^{-2}$ \\
\hline Distilled water & $1.005 \times 10^{-3}$ & 1000 & $7.275 \times 10^{-2}$ \\
\hline
\end{tabular}

Experiments consisted of systematically setting flow rater and frequencies and observing the resulting droplet stream under the stroboscope to determine if a condition of stable, uniform droplet formation existed. For each combination of urifice size and fluid tesied, frequency and flow rate were adjusted until the entire range of obtainatile frequencies and flow rates for which stable droplet formation was obtained. The minimum operable frequency, a lower pinch-point, was the starting point of all experiments on a particular combinatic7 of orifice and fluid. In the ethylene glyco! runs, flow rate was inc:eased stepwise and the maximum and minimum frequencies for good droplet formation were found at each step. Step size was chosen to give 8 to 16 data points (minimum and maximum) before the pressure limitations of the equipment $(50 \mathrm{~cm} \mathrm{Hg})$ were reached. On the other hand, in the water and 20\%-aqueous glycerol runs, frequency was increased stepwise and the minimum and maximum flow rates for good droplet formation were found $a^{t}$. each step.

At the upper and lower bounds of each step, measurements of $n$. nnometer pressure, feed-tank level, amplifier vol tage, current and frequency, droplet spacing, and length and position of good droplet region within the jet were recorded. Droplet spacing was determined by positioning a ruler behind the droplet stream and counting the number of droplets in one inch of jet length. In the experiments using ethylene glycol, the flow rate was measured directly for each run by diverting the drcplet stream into a beaker for a measured time and weighing its contents. Calibration plots of flow rate versus pressure upstream of the nozzle were driveloped by the same flow measurement technique for water and 20\%-aqueous glycerol for all the nozzles used (see Appendix 9.3). Flow rates in the water and 20\%-aqueous glycerol experiments were read directly from these plots.

In the 67 experiments with water, all four nozzles were investigated; while in the 25 glycol and $2520 \%$-aqueous glycerol experiments, time constraints permitted investigation with only the three smaller nozzles $(325-500 \mathrm{~mm})$. These resulted in 117 data points to be ised in the correlations. 


\subsubsection{Catcher-Fluid Stream}

Several factor: can affect the performance of the catcher streams: catcher-stream velocity, choice of catcher fluid, ratio of catcher-stream velocity to droplet-stream velocity, angle between catcher stream and droplet stream, and shape of catcher-stream nozzle. In this study a single catcher fluid (TCE) and a single catcher-stream nozzle (see Fig. 3B) were investigated. At velocities up to the maximum obtainable on the equipment $(80 \mathrm{~cm} / \mathrm{s})$, no uniform catcher stream could be formed; ripples always appeared in the surface of the stream.

To form a more uniform catcher-stream surface, the stream was directed onto a 15-cm squąr? Pyrex glass plate. For the two flow rates investigated ( 7.8 and $\left.10.1 \mathrm{~cm}^{3} ; \mathrm{s}\right)$, the angle of incidence of the catcher stream upon the glass was varied from 0 to $180^{\circ}$, and the uniformity of the catcher straam surface was observed.

\section{RESULTS}

\subsection{Experimental Observations}

In the experiments, the behavior of ahout $22 \mathrm{~cm}$ of the jet was observed. Figures 5 through 7 are a series of photographs taken for a water jet issuing from a 350-um nozzle. Figure 5 shows a natural jet which breaks up into droplets of various diameters. The coherent portion of the jet is $4 \mathrm{~cm}$. Droplet spacing is nonuniform, and coalescence of droplets appears throughout the jet. In Figs. 6 and 7, a frequency of $800 \mathrm{~Hz}$ was imposed on the nozzle. Within a certain power range, a steady jet breaks into uniform droplets. At steady state the first break in the liquid stream occurs at $1.5 \mathrm{~cm}$ from the orifice. The region of good drops extends to $13 \mathrm{~cm}$, where doublets begin to appear. When excessive power is applied, causing a large amplitude of vibration, the jet disintegrates into several streams (atomization), which can be seen in Fig. 7 .

Droplet diameters are correspondingly larger, and more details of the drops are observed when a larger nozzle is used. Figure 8 shows a water jet issuing from a $501-\mu \mathrm{m}$ nozzle with an imposed frequency of $1000 \mathrm{~Hz}$. The photograph shows the oscillation and deformation of droplets caused by the waveform of the vibrator. The photographs in Cline and Anthony (4) show similar droplet behavior.

Both forward-merging and rear-merging satellites were observed for almost all runs and for different values of oscillator power. However, forward-merging satellites were more common than the rear-merging variety. Figure 9 is a photograph of a water jet, which shows the appearance of forward-merging satellites. It can also be noticed that the satellite stream is slightly off-center. 


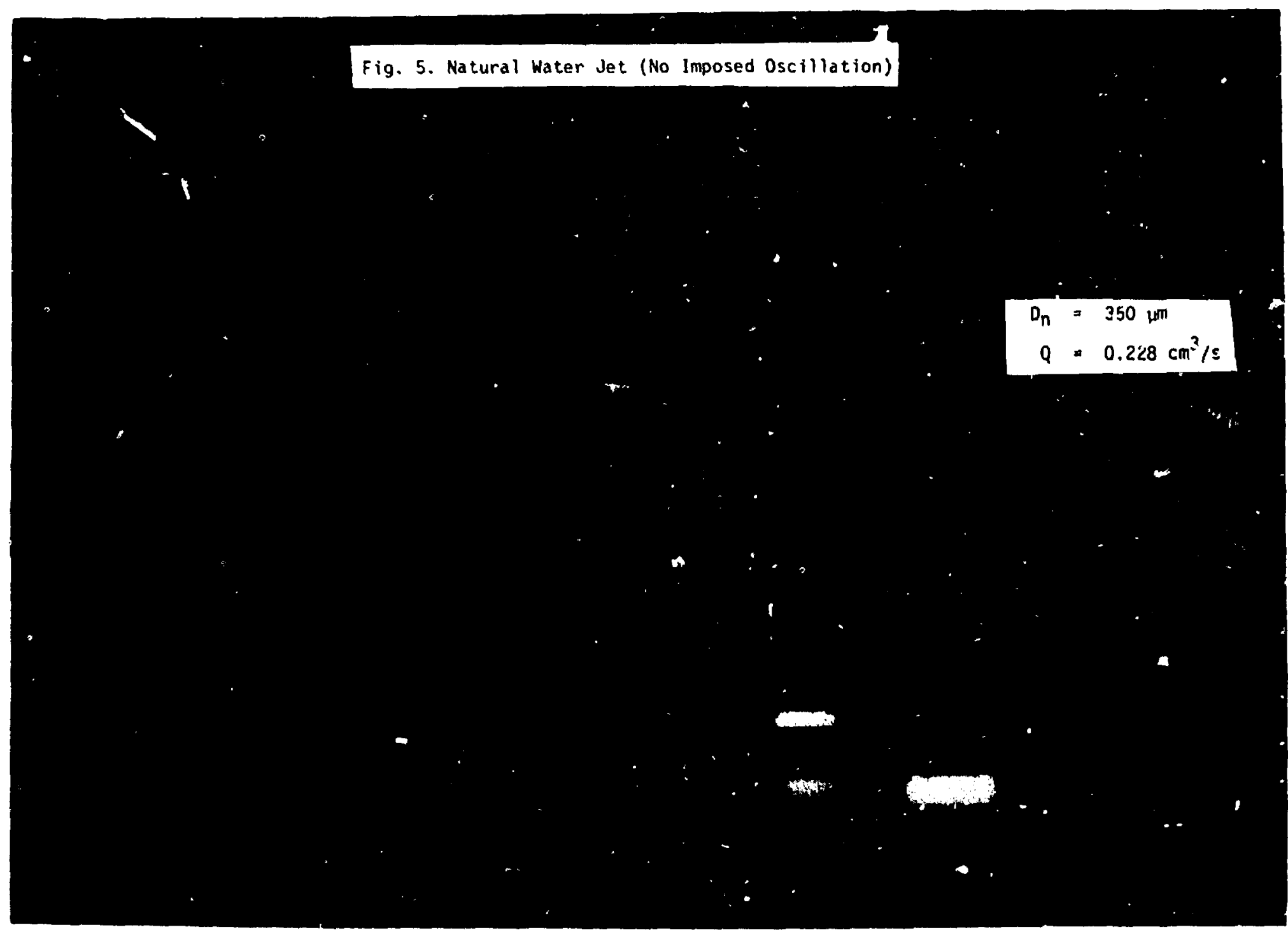


ORNL-Photo 5721-81

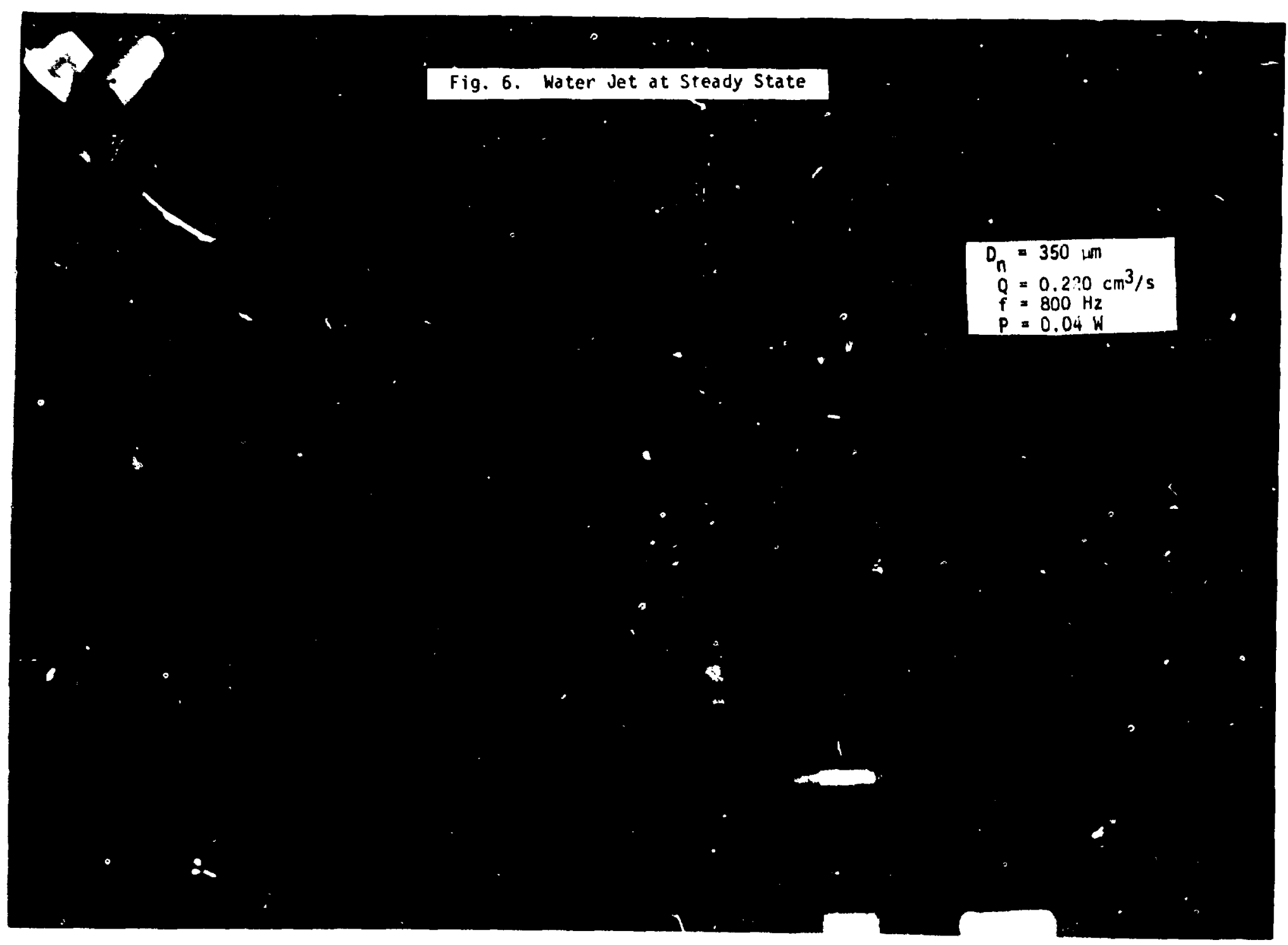


ORNL-Pho to 5722-31

-

\section{Fig. 9. Breakup of a Water Jet with satellite formation}

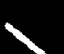
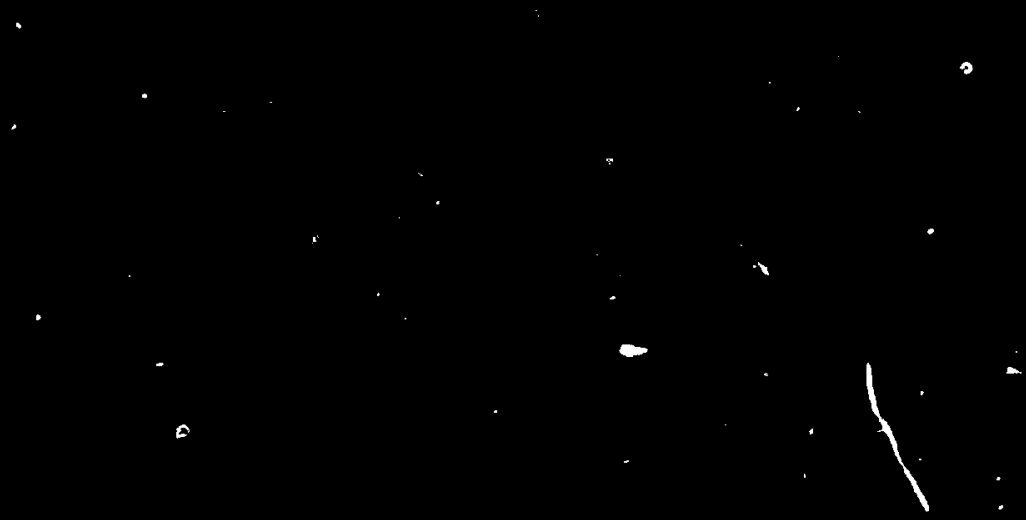

0 
ORNL-Photo 5725-81

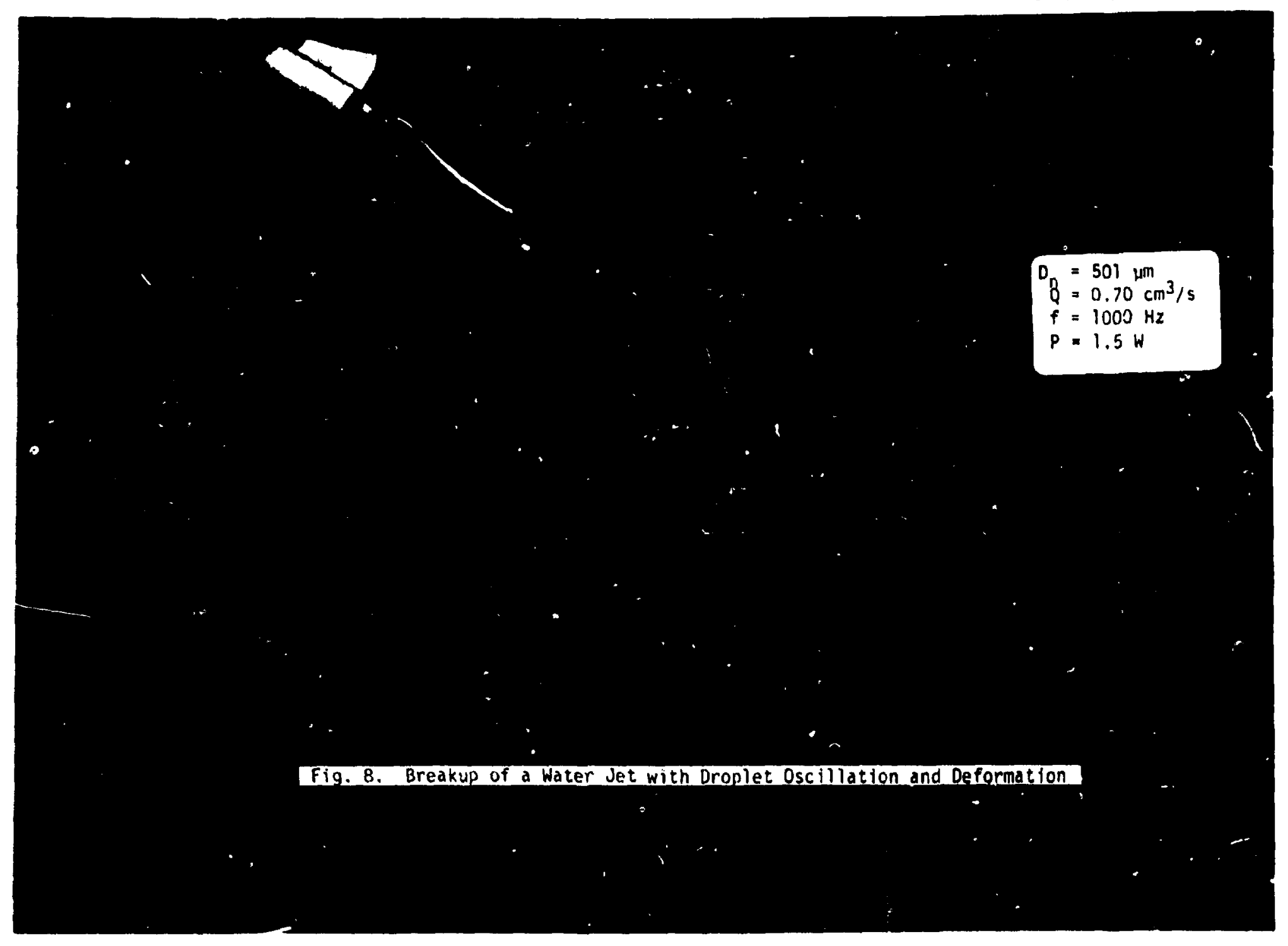


ORNL-Photo 5724-81

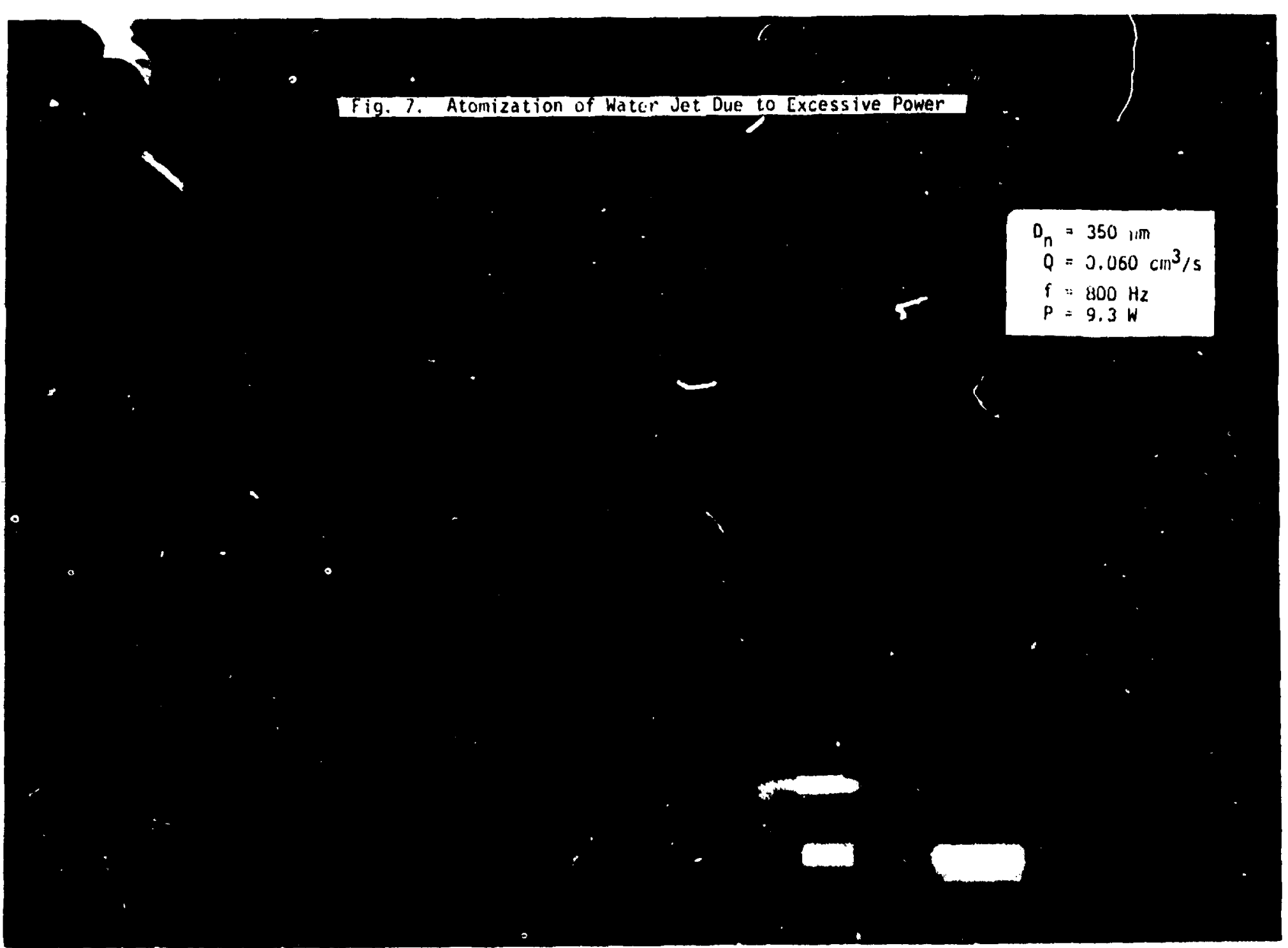


Sixty run: were made to determine the minimum fluid flow rate allowable at a given frequency. For each fluid and a particular nozzle, these minimum flow rates, c: equivalently, minimum velocities, were then related to the frequencies. Figure 10 is a plot generated in this manner. Each point in Fig. 10 represents an experiment where a stable operation and uniform droplet formation existed. Similariy, other combinations of nozzles and fluids were examired. Furthermore, another 54 runs were made to determine the maximum flow rate for a given frequency. Figure 11 shows all data points for the minimum case, and Figure 12 summarizes runs at maximum flow rates.

The data obtained indicated that two correlations are necessary to fully describe the upper and lower bounds of good droplet formation. The upper bound is the maximum flow rate at a fixed frequency, which produces good drop formation. Equivalently, the upper bound is the minimum frequency at a fixed flow rate, with all else being constant, which will form good Jrops. Physically, excessive flow rate and low frequency causes more fluid to be available than is required for single-drop formation. Hence, the drop disintegrates and forms side streams. Similarly, the lower bound for uniform droplet formation is the minimum flow rate at a fixed frequency attainable if the flow rate is fixed. The droplet diameter as well as the void spacing between the drops are excessively small, causing coalescence to occur.

\subsection{Dimensional Analysis}

The problem of selecting parameters to enter into the correlations can be solved by a dimensional analysis, because this will combine parameters into meaningful dimensionless groups. In this analysis, the operable frequency is assumed to be a function of viscosity, surface tension, density, nozzle diameter, gravitational force, dropiet spacing, and power output of the amplifier. The actual amplitude of the displacement of the vibrator diaphragm may be an important variable, but it is assumed here that the diaphragm displacement is linearly proportional to the power supplied to the vibrator. The dimensional analysis has an infinite number cf possible solutions (see Áppendix 9.4). However, for all cases examined, five important dimensionless groups emerged: Reynolds, Weber, and Froude numbers, a Power number $P_{0}$, and a dimensioniess droplet spacing. The droplet spacing $\lambda$ is defined as the center-to-center distance of the main droplets. The result of dimensional analysis is given as:

$$
\left(\frac{\lambda}{D_{n}}\right)=F^{\prime}(\operatorname{Re})^{A}(W e)^{B}(F r)^{C}\left(P_{0}\right)^{D}
$$

where

$$
F^{\prime}=\text { constani }
$$




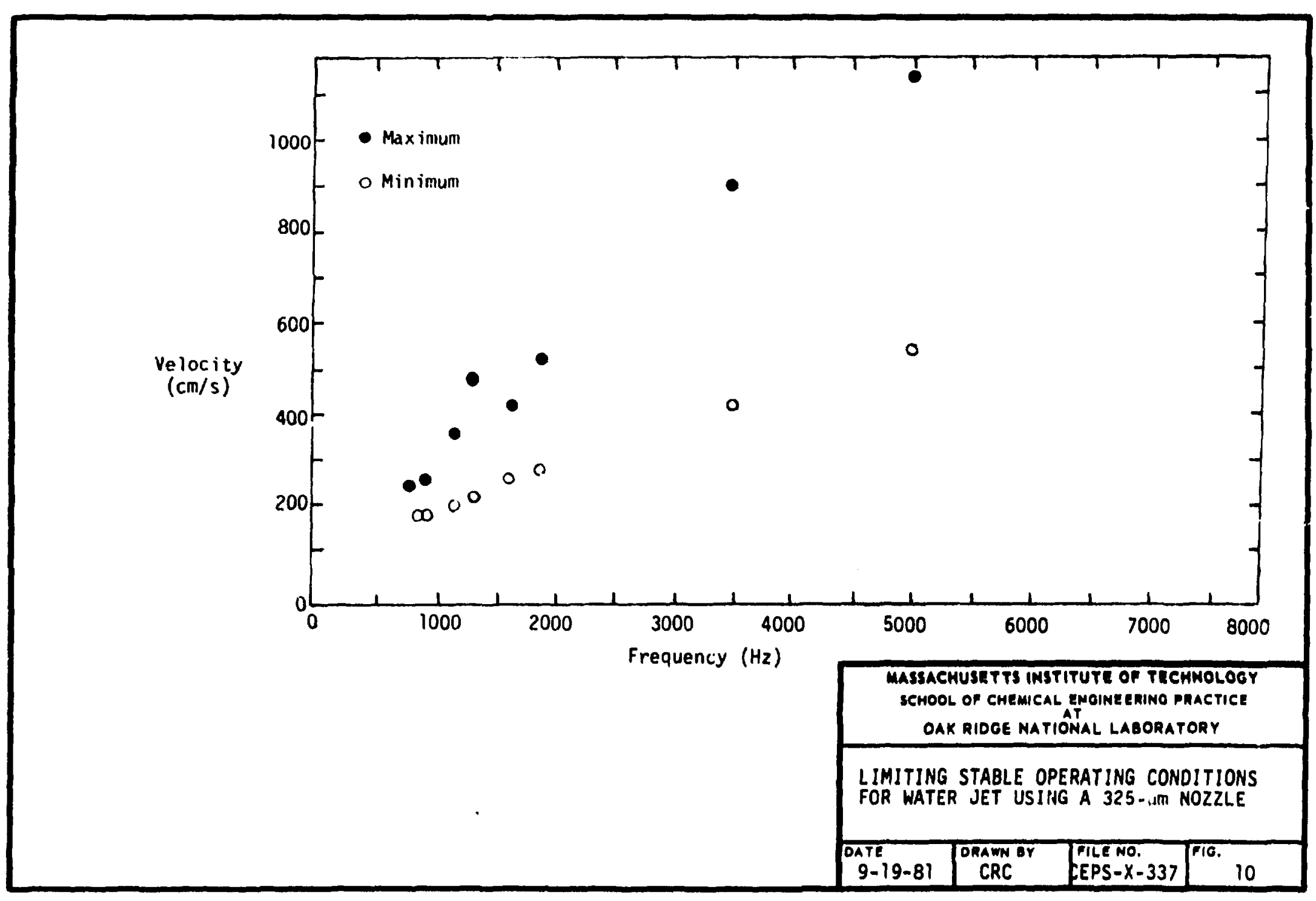




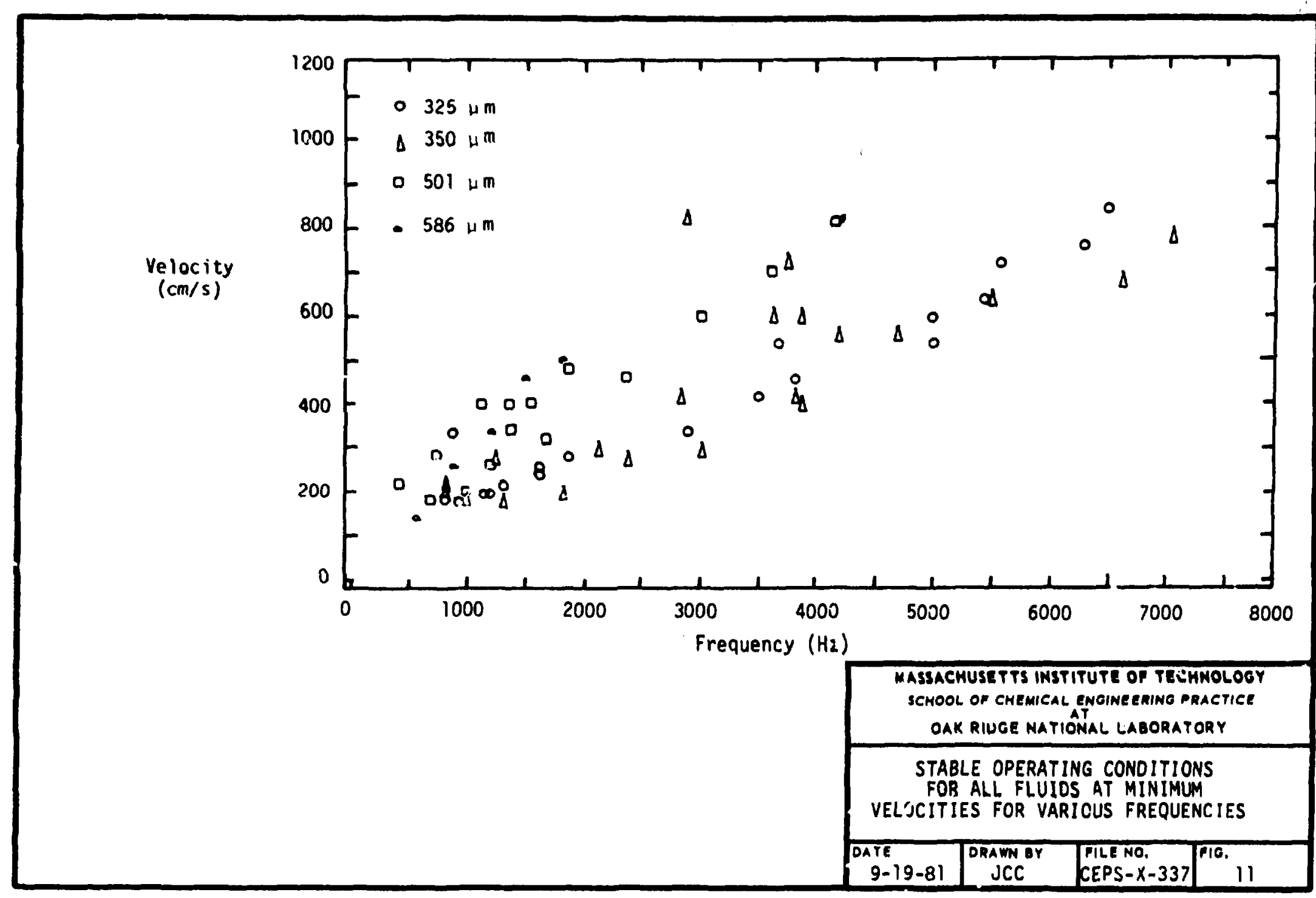




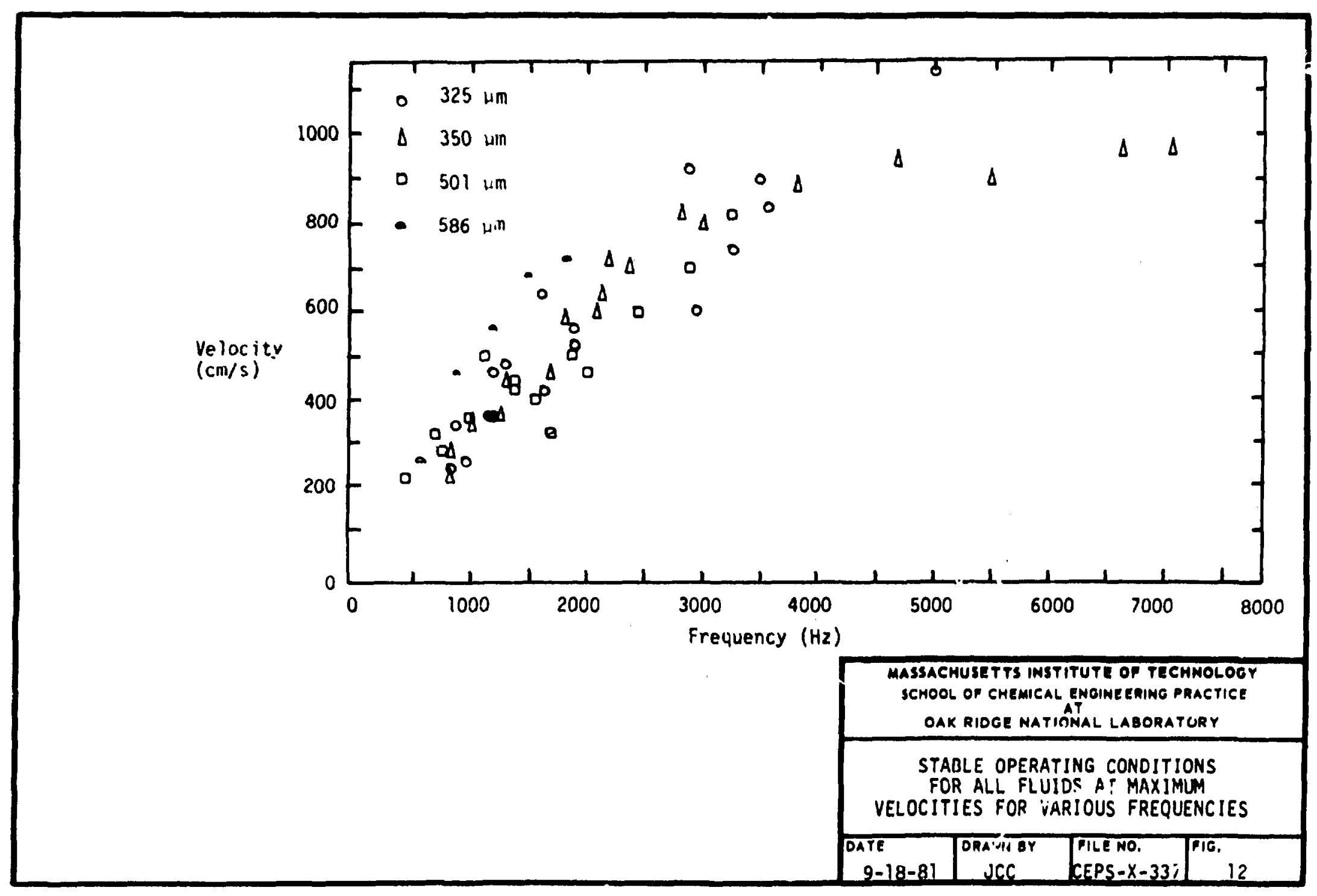




$$
\begin{aligned}
& \frac{\lambda}{D_{n}}=\text { dimensionless droplet spacing } \\
& R e=\frac{D_{n} v \rho}{\mu}=\text { Reynolds Number } \\
& \text { He }=\frac{C_{n} v^{2} \rho}{\sigma}=\text { Hcoel Number } \\
& F r=\frac{v^{2}}{g D_{n}}=\text { Froude Number } \\
& P_{0}=\frac{p}{\rho f^{3} \lambda^{2} D_{n}^{3}}=\text { Power Number }
\end{aligned}
$$

\subsection{Computer Models}

With the aid of SAS (Statistical Analysis System) programs (15), a correlation was developed for the upper-bound case, and a similar relationship was developed for the lower-bound case. These are shown in Table 3.

The model for the upper bound case indicates that dependence on Reynolds number and Power number is negiigible, while Weber and Froude

numbers are more important. The model for the iuwer bound, however, shows that all diriensionless groups are about equally insignificant. The model practically gives a constant value of $\lambda / D$. The table has also listed the values of $\hat{R}^{2}$ and the coefficient of variation. $R^{2}$ measures how much variation in the dependent variable can be sccounted for by the model. It can range from 0 (no correlation) to 1 (perfect correlation). Coefficient of variation describes the amount of variation in the population. Appendix 9.5 contains a listing of the program, which was written to access the SAS routines and to perform the data regression.

\subsection{Catcher-Fluid Stream}

When trichloroethylene (TCE) was intraduced through the $500 . \mu \mathrm{m} \mathrm{slot}$, the liquid sheet contained nonuniformities in the form of ripples. The liquid sheet collapsed into a single thread within the first inch. When the liquid sheet was directed onto a $15 \times 15-\mathrm{cm}$ Pyrex glass plate, the liquid sheet stayed coherent, although it spread and became thinner. The surfare of this moving liquid film was more uniform than before. 
Table 3. Models for the Upper and Lower Bounds of Stable and Uniform Droplet Formation

Model for the Upper Bound

$$
\begin{aligned}
\left(\frac{\lambda}{D_{n}}\right)=1.66 \mathrm{Re}^{-0.00043} \mathrm{He}^{-0.43} \mathrm{Fr}^{0.38} \mathrm{Po}^{-0.037} \\
R^{2}=0.52 \\
\text { C.V. }=9.6 \% \\
\left(\frac{\lambda}{D_{n}}\right)_{\text {mean }}=7.54
\end{aligned}
$$

Model for the Lower Bound

$$
\begin{aligned}
\left(\frac{\lambda}{D_{n}}\right)=7.32 & \operatorname{Re}^{0.016} \mathrm{We}^{-0.066} \mathrm{Fr}^{-0.049} \mathrm{PO}^{-0.020} \\
R^{2} & =0.15 \\
\text { C.V. } & =17 \% \\
\left(\frac{\lambda}{D_{n}}\right)_{\text {mean }} & =4.83
\end{aligned}
$$




\section{DISCUSSION}

\subsection{Limitations of the Correlations}

Figures 13 and 14 are the predicted $\ln \left(\lambda / D_{n}\right)$ vs actual $\ln \left(\lambda / D_{n}\right)$ for the upper-and lower-bound cases, respectively. The $45^{\circ}$ straight line represents a perfect fit. The scattering of the data points is random indicating that no systematic bias exists in the models. These models conitin five adjustable constants, and the models are empiric. 1 in nature. Judging from the values of $R^{2}$ and the coefficients of variations (C.V.) for both models as shown in Table 3, the model for the upper bound is more successful than the model for the lower bound in terms of fitting the data. The latter predicts an approximately constant value of $\lambda / D_{n}$, regardless of the values of all four dimensionless groups. Both models should only be applied within the range of operating conditions investigated in this study. Extrapolation is not recommended.

Theoretically, the models can be used to predict the operable range of a parameter once everything else has been specified. Ir practice, however, the application of the model is not straightforward because several variables, such as the nozzle diameter and the velocity, appear more than once in the correlations. Typically, one may want to know the limiting velocities for given $D_{n}, f$, and fluid properties. By definitions, velocity is included in three dimensionless groups, $R e$, We, and $\mathrm{Fr}$, and can therefore be solved by trial-and-error only.

To overcome this difficulty, it is suggested that figs. 11 and 12 should be used directly for a first estimation of the limiting velozities. Within the range of fluid properties tested, the minimum and maximum velocities can be obtained if the frequency is known. Furthermore, linear fits for the maximum and minimum velocities vs frequency are given in Table 4. These linear relationships are entirely empirical, based on recorded values of $f$ and calculated values of $V$. Appropriate units must be used in both equations as shown in Table 4 . It is interresting to notice that these best line fits are quite satisiactory. The values of $R^{2}$ are greater than 0.7 , which are better than the fits obtained for both the upper-bound and lower-bound models. When these best line fits are drawn, as shown in Fig. 15, a "pocket" of allowable operation is thus defined to be the region between the two lines. It is aga in empinasized that the linear relations should not be extrapolated beyond the ranges of parameters investigated. Table 5 summarizes the ranges of several variables.

It is also assumed that one drop is formed por cycle of vibration per orifice (16), and therefore $V=f \lambda$. This is an approximation at best. The acceleration of gravity changes $V$, and the drop spacing changes with distance from the nozzle. However, only the first $22 \mathrm{~cm}$ of the jet was examined in any run. Gravity was relatively unimportant in this region. Furthermore, the calculated droplet spacing $\lambda$ confirmed with the observed $\lambda$ to within 10\%; so the assumption of $V=f \lambda$ was acceptable. 


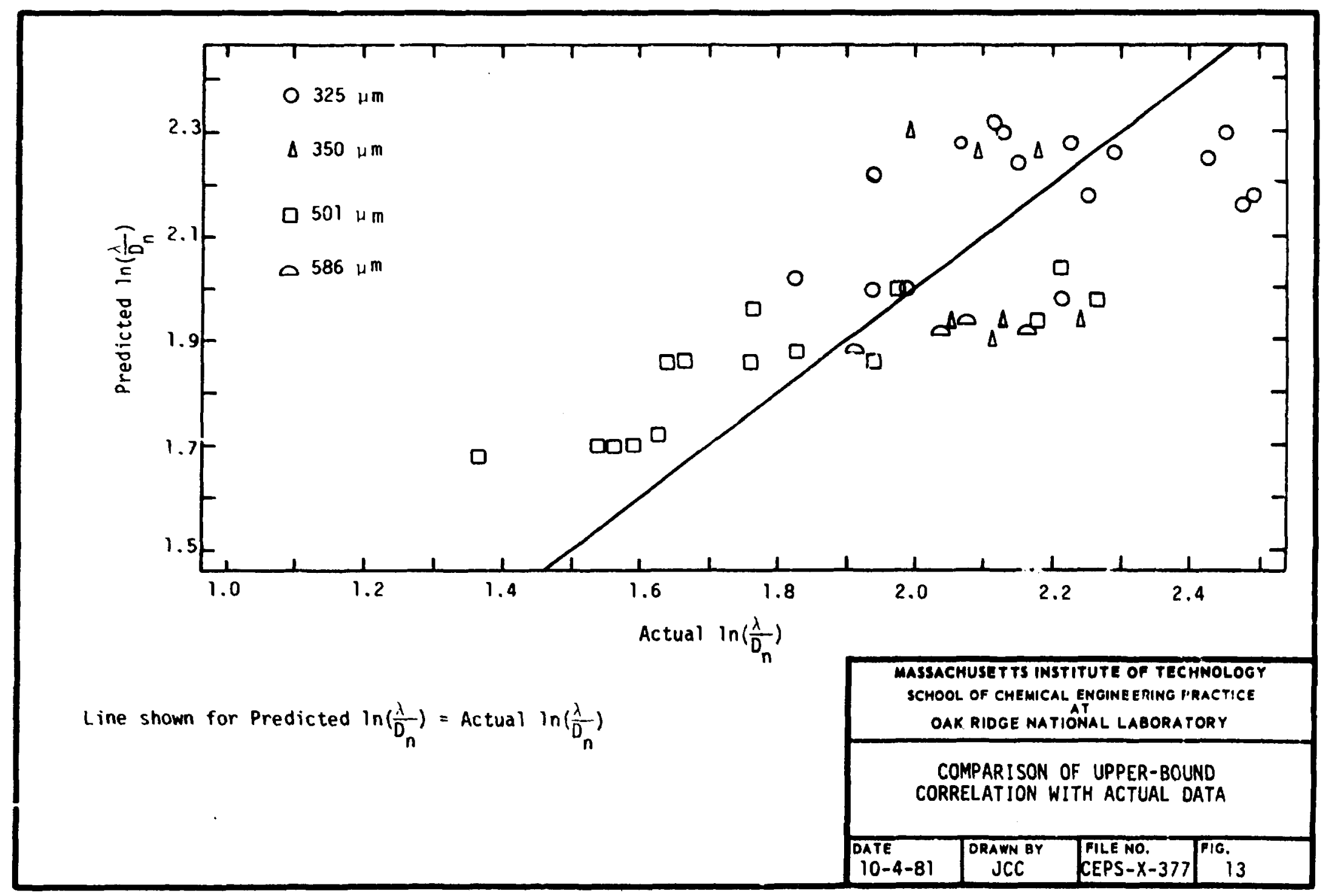




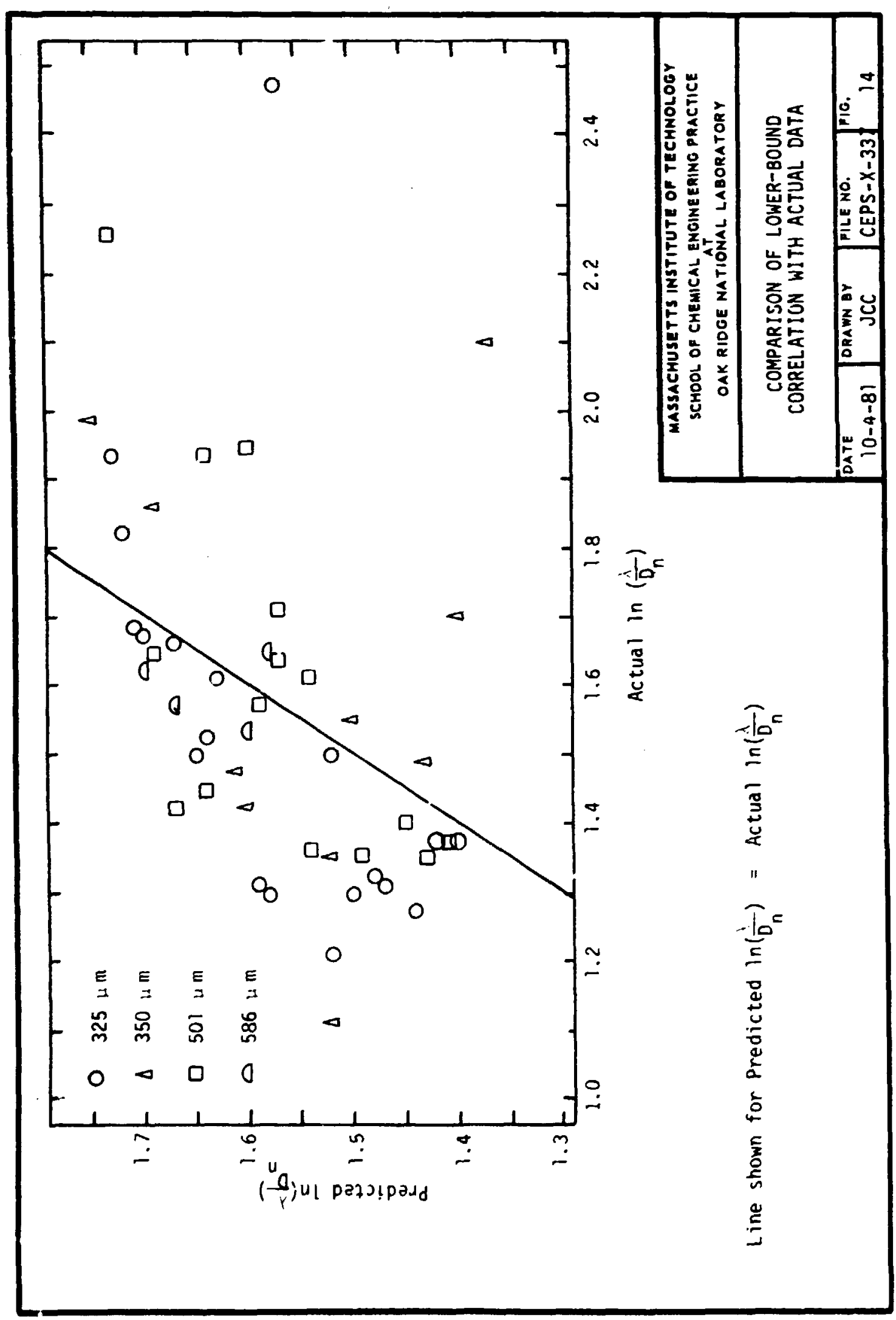


Table 4. Linear-Regression Result for Maximum and Minimum Velocities

$$
\begin{aligned}
V_{\max } & =0.14 f+267 \\
R^{2} & =0.75 \\
\text { C.V. } & =20.8 \% \\
V_{\text {min }} & =0.094 \mathrm{f}+169 \\
R^{2} & =0.72 \\
\text { C. } V .= & 25.6 \% \\
\text { Inits: } V & =\mathrm{cm}^{3} / \mathrm{s} \\
f & =\mathrm{Hz}
\end{aligned}
$$




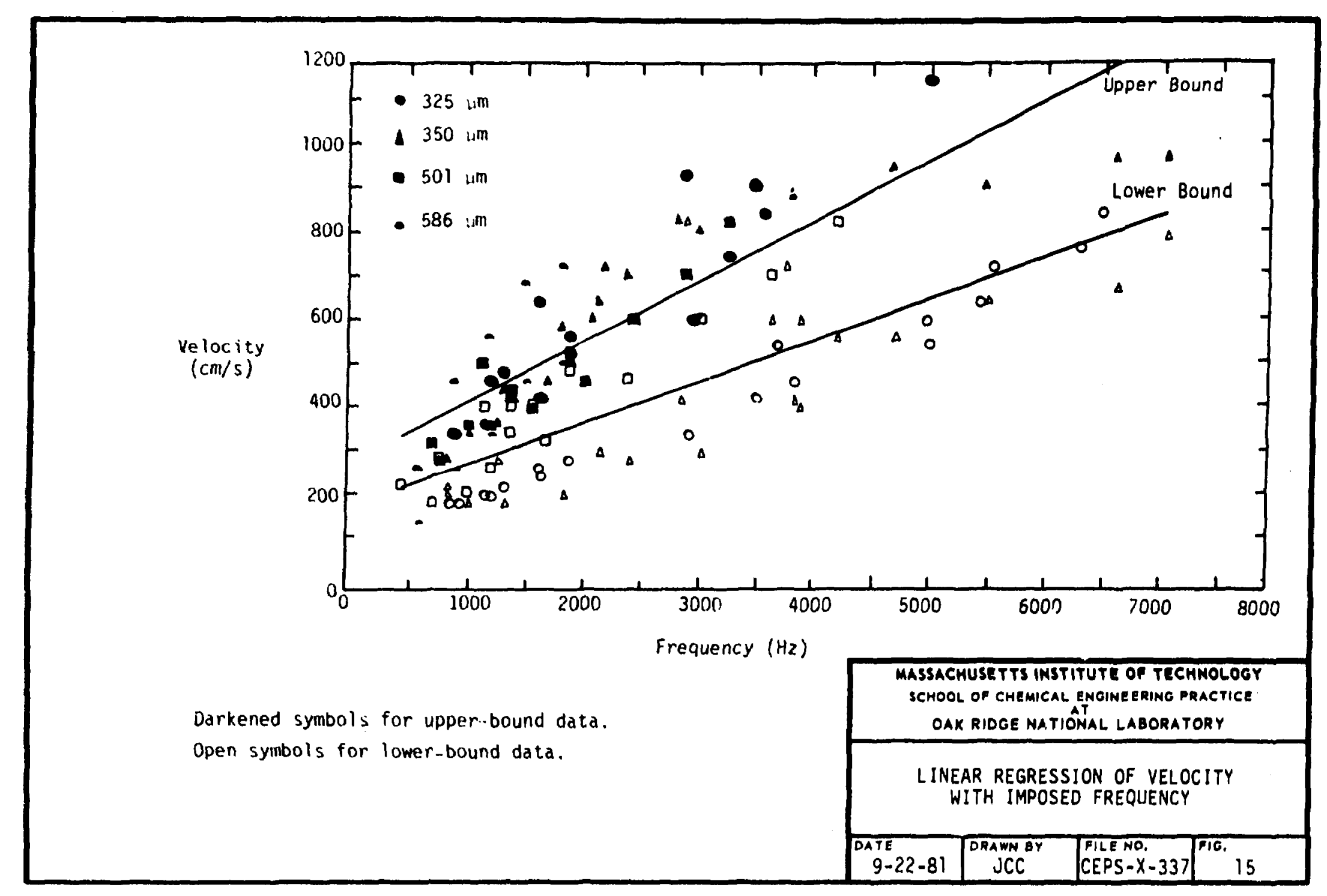

$\tilde{\infty}$ 
Table 5. Ranges of Parameters Investigated

\begin{tabular}{|c|c|c|c|}
\hline & Minimum & Maximum & Possible Error \\
\hline \multicolumn{4}{|l|}{ Fluid Properties (at $20^{\circ} \mathrm{C}$ ) } \\
\hline Density, $p\left(\mathrm{~kg} / \mathrm{m}^{3}\right)$ & 1000 & 1113 & \\
\hline Viscosity, $\mu(\mathrm{Pa}-\mathrm{s})$ & $1.005 \times 10^{-3}$ & $1.99 \times 10^{-2}$ & \\
\hline Surface Tension, $\sigma(N / m)$ & $4.77 \times 10^{-2}$ & $7.275 \times 10^{-2}$ & \\
\hline \multicolumn{4}{|l|}{ Measured Quantities } \\
\hline Frequency, $f(\mathrm{~Hz})$ & 460 & 7050 & \pm 0.1 \\
\hline Nozzle Diameter, $D_{n}(\mu \mathrm{m})$ & 325 & 586 & \pm 14 \\
\hline Flow Rate, Q $\left(\mathrm{cm}^{3} / \mathrm{s}\right)$ & 0.000 & 1.63 & \pm 0.03 \\
\hline Droplet Spacing, $\lambda_{\text {obs }}(\mathrm{mm})$ & 1.016 & 5.080 & $\pm 1 \mathrm{drop} / 2.5 \mathrm{~cm}$ \\
\hline Amp 1 ifier Current, I (amp) & 0 & 1.5 & \pm 0.05 \\
\hline Amplifier Voltage, $E(V)$ & 0 & 6.2 & \pm 0.1 \\
\hline \multicolumn{4}{|l|}{ Derived Quantities } \\
\hline Velocity, V $(\mathrm{cm} / \mathrm{s})$ & 179 & 1133 & \\
\hline Droplet Spacing, $\lambda_{\text {calc }}(m)$ & 1.024 & 5.067 & \\
\hline Droplet Diameter, $O(\mu \mathrm{m})$ & 545 & 1199 & \\
\hline Amplifier Power, $P(W)$ & 0 & 9.3 & \\
\hline \multicolumn{4}{|l|}{ Dimensionless Groups } \\
\hline Reynolds No., Re & 77 & 3664 & \\
\hline Weber No., We & 15 & 804 & \\
\hline Froude No., $\mathrm{Fr}$ & 702 & 40,300 & \\
\hline Power No., Po & $3.96 \times 10^{-7}$ & $2.03 \times 10^{-2}$ & \\
\hline $\begin{array}{l}\text { Dimensionless Droplet } \\
\text { Spacing, } \lambda / D_{n}\end{array}$ & 2.5 & 12 & \\
\hline Diameter Ratio, $D / D_{n}$ & 1.56 & 2.62 & \\
\hline
\end{tabular}




\subsection{Comparison with the Literature}

Table 5 shows that the droplet diameter-to-nozzle diameter ratio $D / D_{n}$ was within the range of 1.56 to 2.62. This agreed with Merrington and Richardson results (12).

The dimensionless droplet spacing for the maximum-flow-rate case was found to have a mean value of 7.54; for the minimum flow rate, the mean value for $\lambda / D_{n}$ was 4.83. Pimbley and Lee $(13)$ concluded that it was least likely to have satellite formation if $\lambda / D_{n}$ fell between 5 and 7 . The data obtained confirmed their findings.

\section{CONCLUSIONS}

1. A set of data was obtained which define the region of good droplet formation over a wide range of fluid properties and operating conditions.

2. Correlations of these data in the form of upper and lower bounds of good droplet formation have been developed.

3. A unique catcher-stream design which can be used with trichloroethylene (TCE) was devised. The TCE remained as a moving liquid film wi thout collapsing into a single thread.

4. Experimental oiservations of dimensionless droplet spacing and ratio of droplet-to-nozzle diameter were found to agree well with previous studies.

\section{RECOMMENDATIONS}

1. The computer-derived correlations should be tested with data obtained from the internal-gelation-process pilot plant in Bidg. 354l, ORNL.

2. The catcher-stream design should be further tested and modified.

3. Develop a physical model for the droplet formation process.

\section{ACKNOWLEDGMENTS}

The authors thank Paul Haac, Al Ryon, and Walt Bond for their guidance. Special thanks are given to Vic Fowler, Rex Moody, and Wiley Johnston for the ir technical assistance. 


\section{APPENDIX}

\subsection{Newtonian-Jet Breakup}

Theories have been established (6) to predict the breakup length of low-speed laminar jets formed by a kewtonian fluid in stagnant air. The breakup length $L$, which is the coherent portion of the jet, is often expressed as a function of jet velocity. The average velocity in the nozzle $V$ is used in almost all correlations because it can be readily obtained. The velocity of the jet surface is zero immediately on emerging from the nozzle. The velocity profile relaxes, and the jet diameter may slightly expand or contract, depending on the fluid properties. For these reasons, the velocity of jet surface is more difficult to define and use in correlations.

The general shape of the breakup curve is shown in Fig. 16. At very low jet velocity, no jet is tu. med, and the liquid drips out of the nozzle. This acccunts for the curved segment $A B C$. The linear portion of the curve (points ACD) was first described by Weber's analys is and later confirmed by others (6). The jet becomes turbulent beyond point $F$ in Fig. 16 . For our investigation, however, only the linear portion ACD will be examined further.

Weber derived the following result:

$$
\frac{L}{D}=C_{1} W^{\frac{1}{2}}(1+3 Z)
$$

where $Z=\mathrm{We}^{\frac{1}{2}} / \mathrm{Re}$, called the Ohnesorge number, was found to be a useful parameter in measuring the relative importance of viscosity and surface tension in controlling stability. It was found that jets are more stable at large Ohnesorge numbers (i.e., high viscosity and low surface tension). The constant $C_{1}$ has an experimental value of 12 (6). Equation (4) is only valid for the linear segment $(A C D)$ on the breakup curve and does not account for the effect of ambient pressure. A modification of Weber's theory, which predicts the entire breakup curve, is available (6). In the same study, two other conclusions are worth mentioning. Over the linear portion of the $L-V$ curve: (1) the first break in the jet, or the breakup length, is always well-defined; (2) the velocity profile of the jet has no effect on the propagation of the disturbar.ce.

There are two minimum physical requirements for droplets to form. First, a certain pressure is necessary before a stream can be emitted from the nozzle. In this case, liquid drips out from the nozzle under gravity force. (The minimum pressure to form a liquid stream is about $1 \mathrm{~cm} / \mathrm{lg}$ for our experimental system. This minimum pressure requirement is always exceeded for all practical purposes.) 


$$
\sqrt{n}
$$


Secondly, there must be enough room in the stream for drops to form. Consider a segment of the stream which would eventually become a single drop. If the frequency is too high or the droplet spacing (which is defined as the center-to-center distance between two drops) is too short, it can be shown that the diameter of the drop that contains this volume of liquid would be larger than the spacing. Droplets will overlap one another, making it impossible to have steady-state drop formation. The wavelength for which the drop diameter just equals the wavelength is given by:

$$
\frac{\pi D_{j}^{2} \lambda}{4}=\frac{\pi}{6} \lambda^{3}
$$

Therefore,

$$
\frac{\lambda}{D_{j}}=\sqrt{n .5}=1.2247
$$

Drop formation is impossible for spacings less thall 1.2247 diameters of the stream.

When the liquid jet breaks into droplets, the fluid spheres oscillate about their equilibrium shape with is characteristic natural frequency. For two low viscosity fluids bounded by a free interface, the natural frequency is given by $(\underline{10}, \underline{11})$ :

$$
B_{I}=B^{*}-\frac{(2 n+1)^{2} \sqrt{\beta^{\star} \mu_{j} \mu_{0} \rho_{j} \rho_{0}}}{2 \sqrt{2} R \Gamma\left[\sqrt{\mu_{j} \rho_{i}}+\sqrt{\mu_{0} \rho_{0}}\right]^{2}}
$$

where

$$
B^{*}=\sqrt{\frac{\sigma(n+1)(n-1)(n+2)}{R^{3} \Gamma}}
$$

and

$$
\Gamma=\rho_{0} n+\rho_{i}(n+1)
$$

Fluid properties, as well as the droplet size, enter into the expression for $\beta_{I}$. The parameter $n$ usually assumes a value of 2 , which is the principle mode of oscillation. Equations (7) through (9) are confirmed to within $10 \%(10,11)$. A drop falling through another fluid is different 
from a solid sphere in that it may be deformed, and it will have a circulation setup within itself by the shearing effect of the relative motion of the two fluids. These are among the initial sources of disturbance which can cause the droplets to oscillate.

Another characteristic parameter for droplets from narrow jets is called the limiting velocity $v_{0}$. Below this 1 imiting velocity and for a given fluid, the drops will be uniform in size. In fact, Merrington and Richardson (12) found that for fine nozzles (below 1-mm diam) the constant drop size reached at low velocities is roughly twice the nozzle diameter. The ratio of droplet diameter $D$ to jet diameter $D_{j}$ has a mean value of 2.1 for nozzle diameters ranging from 0.30 to $0.65 \mathrm{~mm}$. When jets issue from larger nozzles at low speeds, the drops are no longer uniformly twice the diameter of the nozzle. This limiting velocity $v_{0}$ is originally given as:

$$
\frac{\mu}{\sqrt{\rho \sigma D_{n}}}=2000\left(\frac{\mu}{v_{0} \rho D_{n}}\right)^{4 / 3}
$$

Equivalently,

$$
v_{0}=299\left(\frac{\mu^{2} \sigma^{3}}{\rho^{5} D_{n}^{5}}\right)^{1 / 8}
$$

To summarize, some characteristics of a natural Newtonian jet, such as the breakup length, are well-known. There are physical limitations for droplets to form from a jet, such as a minimum pressure and a minimum spacing required. A liquid drop has a characteristic natural frequency of vibraiion when it is disturbed. Also, a limiting velocity exists for droplets from a narrow jet. On the other hand, only a limited amount of literature focused on a liquid jet that has an imposed oscillation. In particular, the factors that affect the uniformity of droplets formed this way is largely unknown.

\subsection{Accurate Calibration Curves}

Calibration curves describing flow rate as a function of pressure upstream of the nozzle were developed for water and $20 \%$ aqueous giycerol for all nozzles. At several different flow rates, the droplet stream was divided into a beaker for a measured time and the contents were weighed. The volumetric flow rate was then calculated from the liquid density, and this measured mass flow rate. A typical example is given in Fig. 17. The remaining curves may be found in the calculation file. 


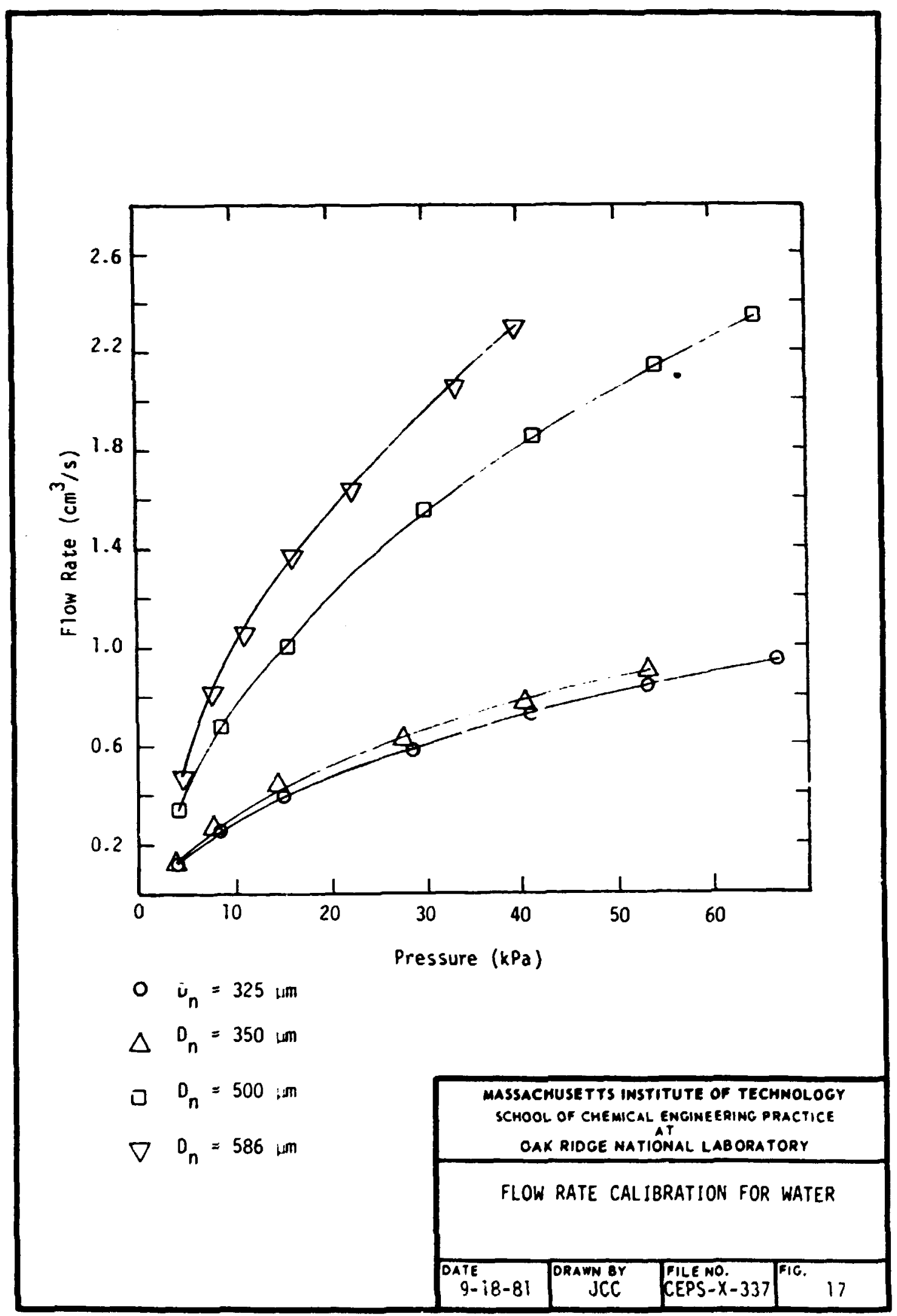




\subsection{Dimensional Analysis}

A dimensional analysis was performed, in which the important dimensionless groups were identified as Reynolds number (Re), Weber number (He), Froude number $(\mathrm{Fr})$, Power number $(\mathrm{Po})$, and droplet spacing-to-nozzle diameter ratio $\left(\lambda / D_{n}\right)$ The vibration frequency was defined as a function of the dimensional parameters as shown below:

$$
f=F\left(\mu, \rho, \sigma, Y, \lambda, D_{n}, F, g\right)
$$

A power relationship was assumed:

$$
f=F(\mu)^{a}(\rho)^{b}(\sigma)^{c}(V)^{d}(\lambda)^{e}\left(D_{n}\right)^{g}(P)^{h}(g)^{k}
$$

and the dimensional units [in terms of mass, length, and time, (MLT)], of each of the parameters were then substituted into Eq. (13). This yielded:

$$
\left(\frac{l}{T}\right)=F\left(\frac{M}{L T}\right)^{a}\left(\frac{M}{L^{3}}\right)^{b}\left(\frac{M}{T^{2}}\right)^{c}\left(\frac{L}{T}\right)^{d}(L)^{e}(L)^{g}\left(\frac{M L}{T^{3}}\right)^{h}\left(\frac{L}{T^{2}}\right)^{k}
$$

Equating exponents of like terms gives:

$$
\begin{aligned}
& L: \quad 0=-a-3 b+d+e+g+2 h+k \\
& M: \quad 0=a+b+c+h \\
& T: \quad-1=-a-2 c-d-3 H-2 k
\end{aligned}
$$

Since there were eight unknowns, but only three equations to relate them, some assumptions were required to solve the system. Since we expect $\mu$, $\sigma, \lambda$, and $P$ to appear in separate numbers, we will derive preferred forms if we do not eliminate the ir exponents $a, c, e$, and $h$. Then,

From Eq. (16): $\quad b=-a-c-h$

From Eq. (17): $\quad d=1-a-2 c-3 h-2 k$

From Eq. (18): $0=-a-3(-a-c-h)+(1-a-2 c-$

$$
3 h-2 k)+e+g+2 h+k
$$


So,

$$
g=-a-c-e-2 h+k-1
$$

Substituting this into Eq. (13) yields:

$$
\begin{array}{r}
f=F(\mu)^{a}(\rho)^{-a-c-h}(\sigma)^{c}(V)^{1-a-2 c-3 h-2 k}(\lambda)^{e} \\
\left(D_{n}\right)^{-a-c-e-2 h+k-1}(P)^{h}(g)^{k}
\end{array}
$$

Grouping terms with like exponents gives:

$$
\left(\frac{f D_{n}}{V}\right)=F\left(\frac{D_{n} V_{p}}{\mu}\right)^{-d}\left(\frac{D_{n} V^{2}}{\sigma}\right)^{-b}\left(\frac{\lambda}{D_{n}}\right)^{e}\left(\frac{p}{\rho v^{3} D_{n}^{2}}\right)^{n}\left(\frac{v^{2}}{D_{n} g}\right)^{-k}
$$

Multiplying both sides by $\left(\frac{\lambda}{D_{n}}\right)^{h}$ and assuming that $V=f \lambda$ gives:

$$
\left(\frac{f D_{n}}{V}\right)\left(\frac{\lambda}{D_{n}}\right)^{h}=F(R e)^{-a}(W e)^{-b}\left(\frac{\lambda}{D_{n}}\right)^{e}(F r)^{-k}\left(\frac{p}{\rho f^{3} \lambda^{2} D_{n}^{3}}\right)^{h}
$$

or,

$$
\left(\frac{\lambda}{D_{n}}\right)^{h-e-1}=F(\operatorname{Re})^{-a}\left(W_{e}\right)^{-b}(F r)^{-k}\left(P_{0}\right)^{h}
$$

Making the left-hand-side exponent equal to unity gives:

$$
\frac{\lambda}{D_{n}}=F^{\prime}(R e)^{\frac{a}{1+e-h}}\left(W_{e}\right)^{\frac{b}{1+e-h}}(F r)^{\frac{k}{1+e-h}}\left(P_{0}\right)^{\frac{-h}{1+e-h}}
$$

Finally,

$$
\frac{\lambda}{D_{n}}=F^{\prime}(\operatorname{Re})^{A}(W e)^{B}(F r)^{C}\left(P_{0}\right)^{D}
$$




\subsection{SAS Program Listing and Data Base}

The following is a listing of the SAS computer program used and its data base:

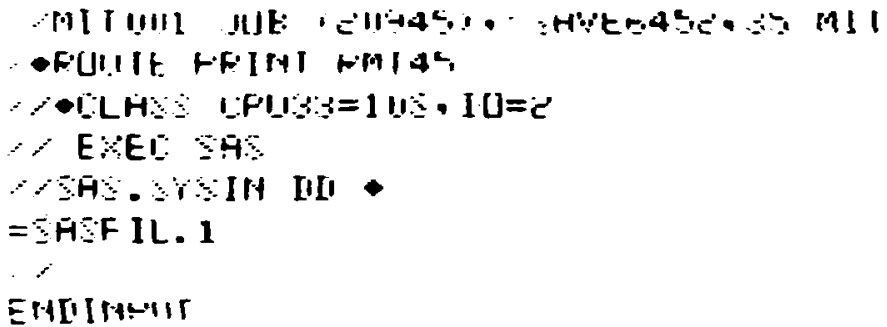

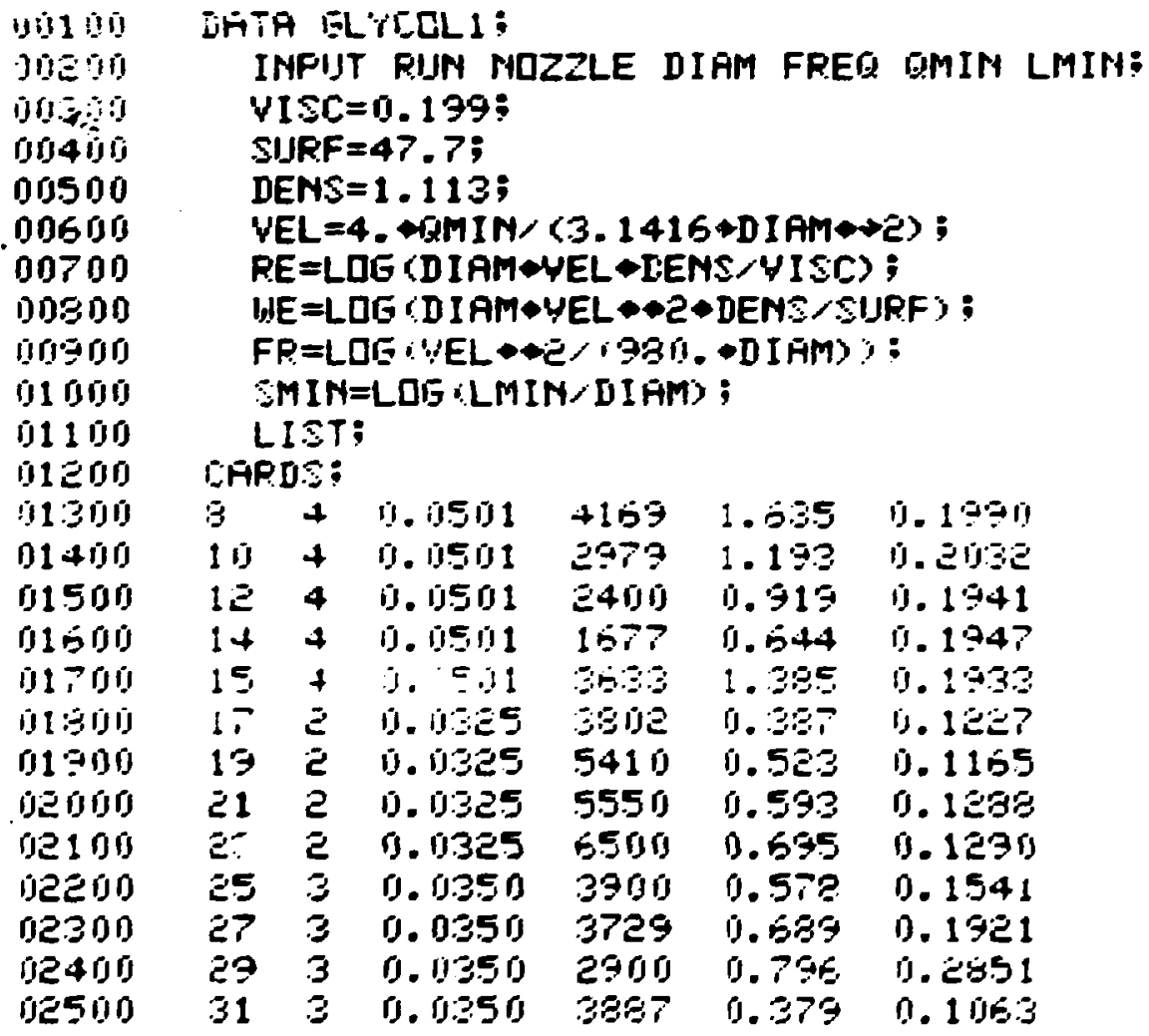




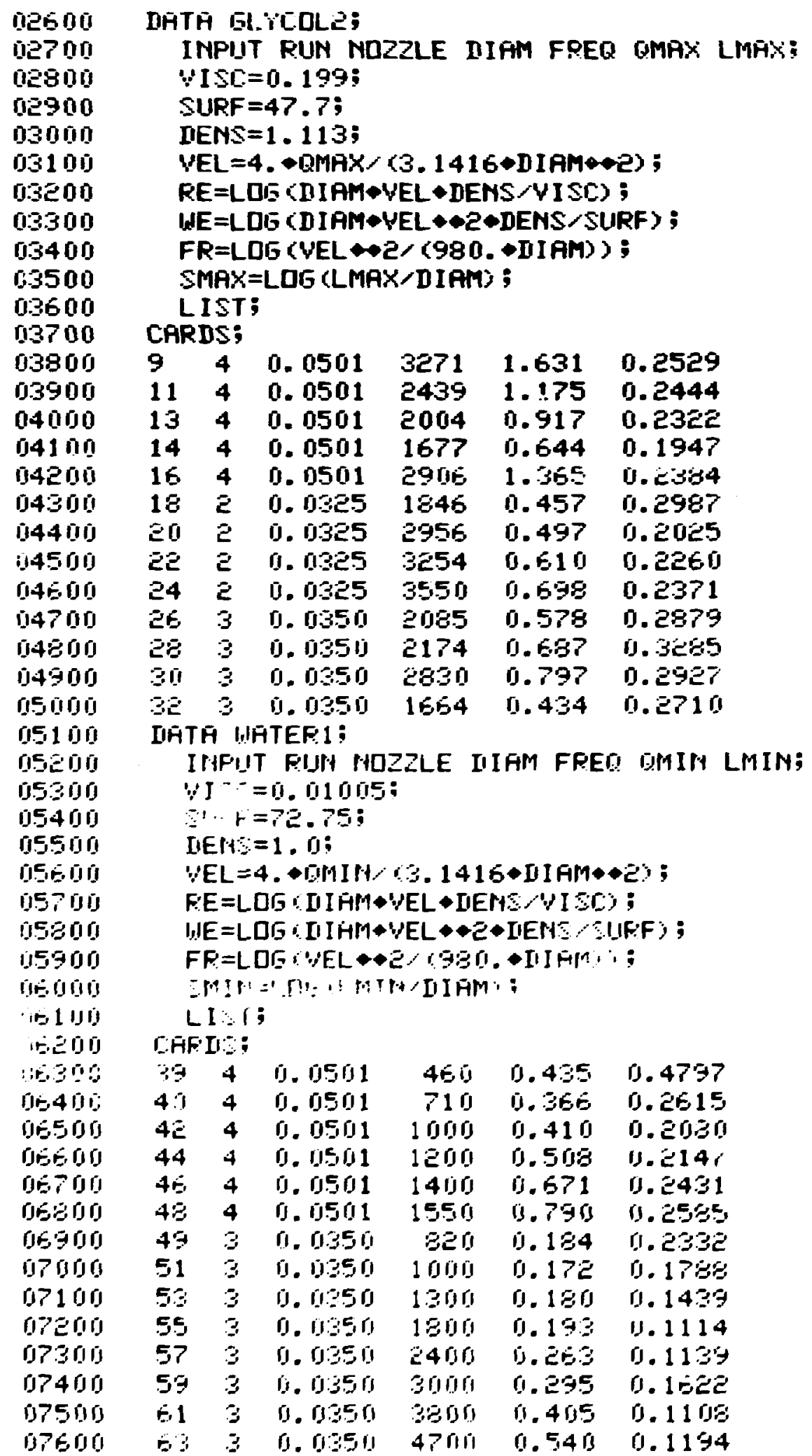




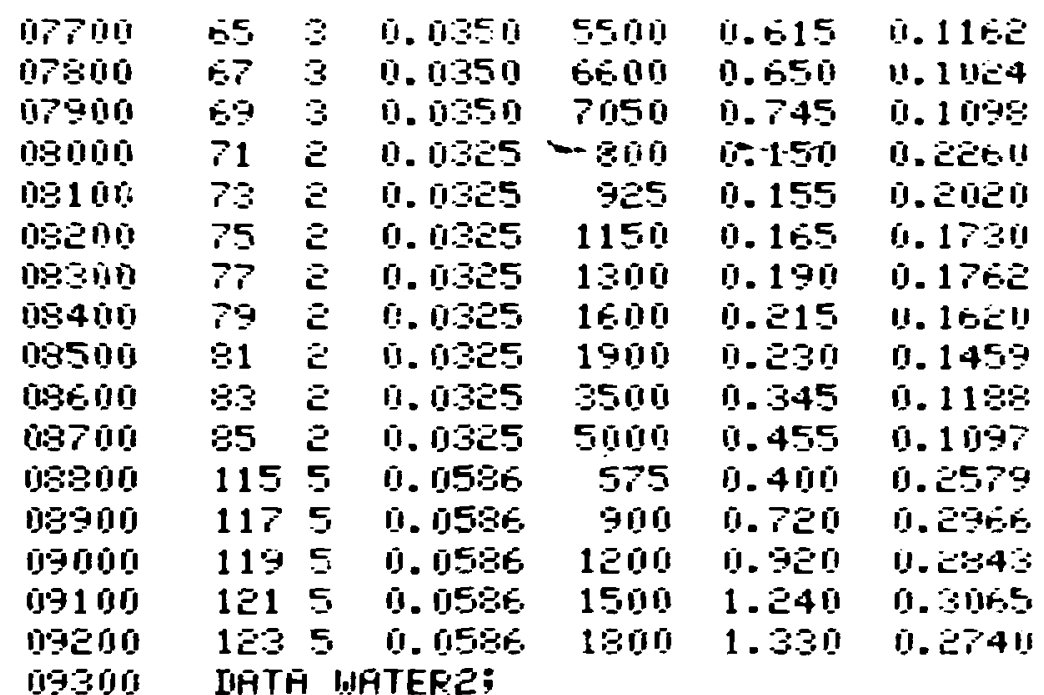

n'940in

095010

IHFIIT FIIM HOZZLE IIAM FFED DNHA LMAX;

$\because \mathrm{ISC}=0.0100 \mathrm{O}$

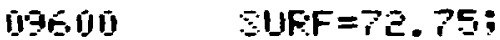

09700

109800

09500

100010

10101

10 वृ!

10300

10400

1050010

1 UJE. III

10700

1 ij:

10900

110000

11100

112000

11300

114010

115010

$11+\operatorname{lin}$

1170

11300

11900

IIEHS $=1$. I0;

$\forall E L=4 . * D M A \%-3.1416+I I A M+\bullet 2) ;$

$F E=L O G$ ODIAM $W E L+I E M S Y I S C ;$

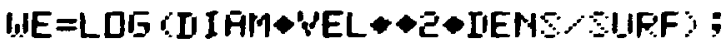

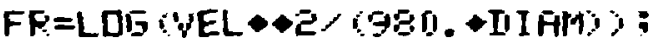

MAF\%=LDS CLMA\% IIIAMY;

LIST;

CAFIS:

\begin{tabular}{|c|c|c|c|c|}
\hline 4 & 0.051 & 400 & 10.435 & 11. \\
\hline 4 & 0.0101 & 710 & 1). 5.40 & \\
\hline 4 & 0.0 .501 & 1000 & !. & \\
\hline 4 & 0. & y & ij & \\
\hline 4 & 0. & 14 & & \\
\hline 4 & 9. & 15.511 & & \\
\hline 3 & ij. & $82 !$ & & \\
\hline$\xi$ & 0. & 10 & & \\
\hline$\xi$ & 0.0350 & 1300 & j. & \\
\hline 3 & 0.050 & 18010 & i. & \\
\hline$\because$ & & 24010 & & \\
\hline 3 & & 30100 & & \\
\hline 3 & & 38 & & \\
\hline 3 & & 47010 & & \\
\hline 3 & & 5.5010 & & \\
\hline 3 & 10. & $6 \in[0]$ & 9. & \\
\hline 3 & 0. & 7050 & & \\
\hline 2 & 0.0 & 800 & 10. & \\
\hline z & 0.0325 & 725 & 0. & \\
\hline$z$ & 0.0325 & 1150 & 0. & \\
\hline 2 & 0.0 & 1300 & I. & \\
\hline 2 & 9.0 & 1600 & 0 & \\
\hline 2 & 0. & .350 & is. & \\
\hline 5 & 9.0 & 5000 & 0.940 & \\
\hline
\end{tabular}




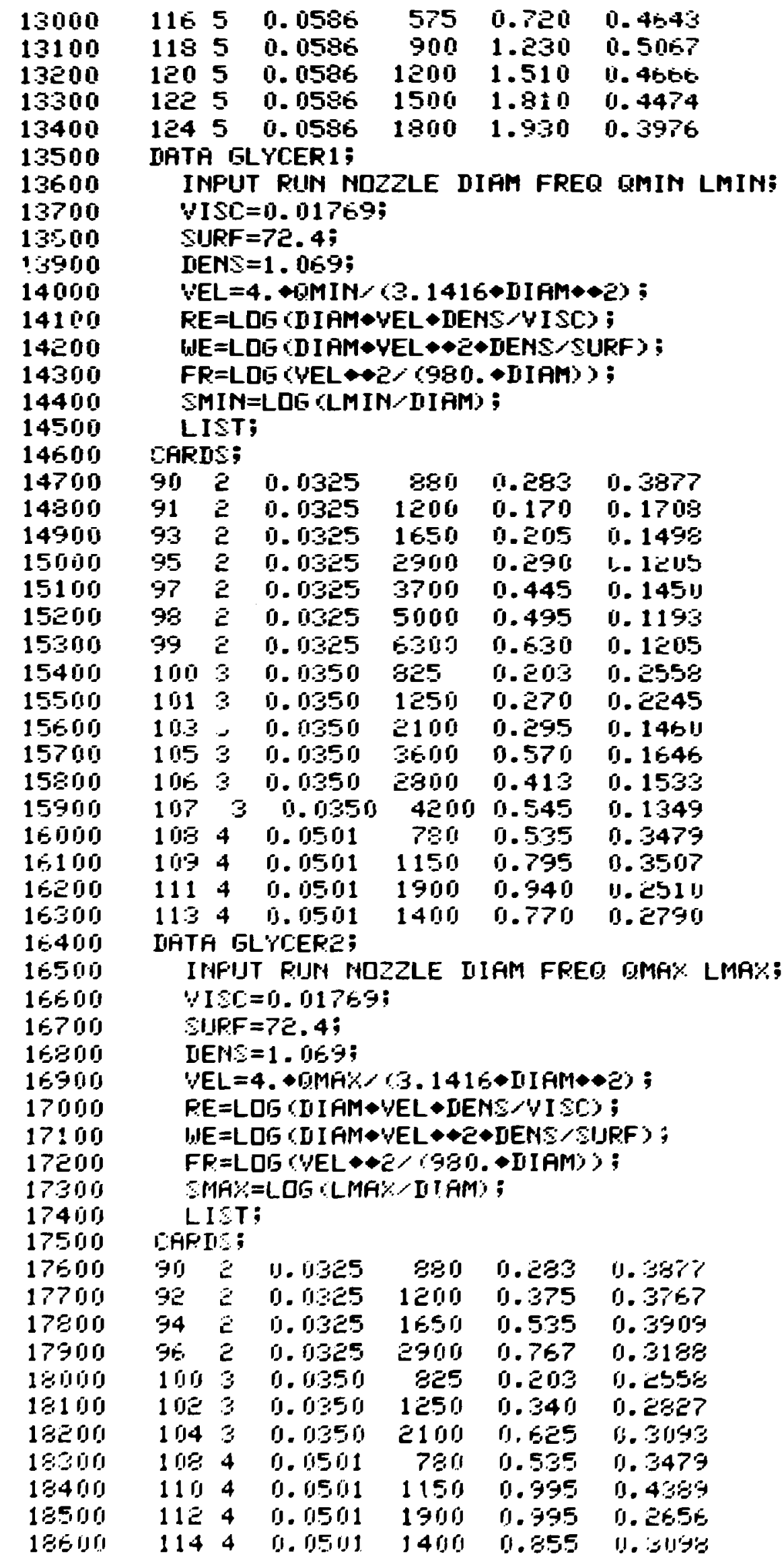




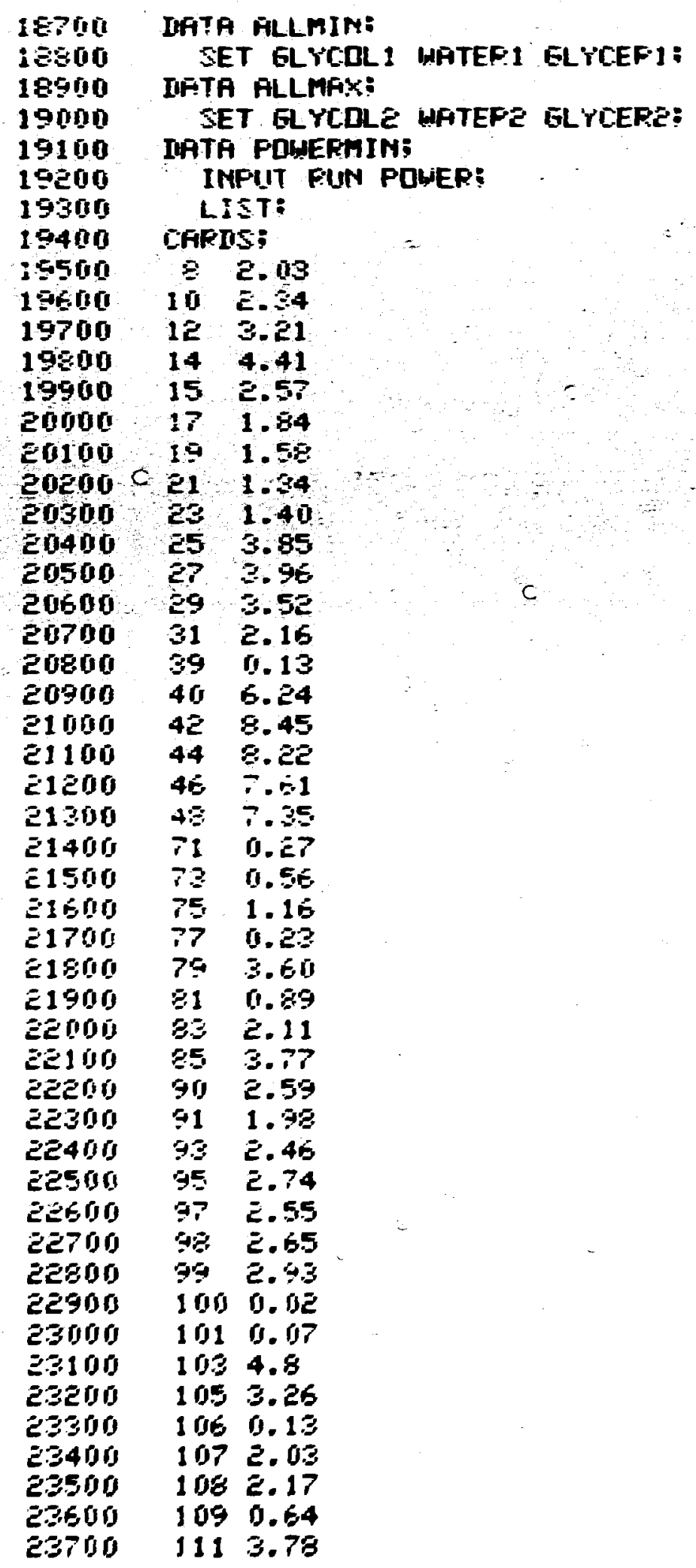




\section{3}

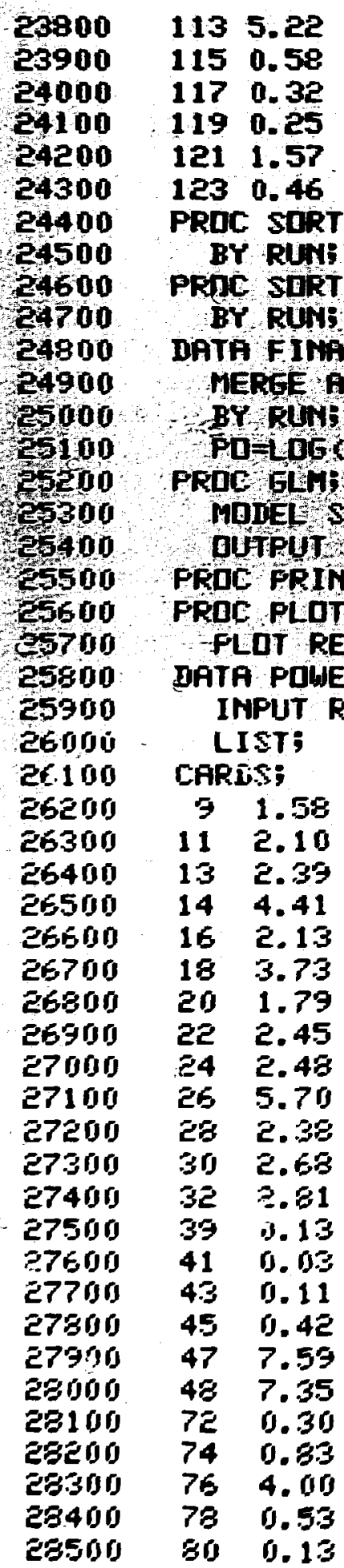




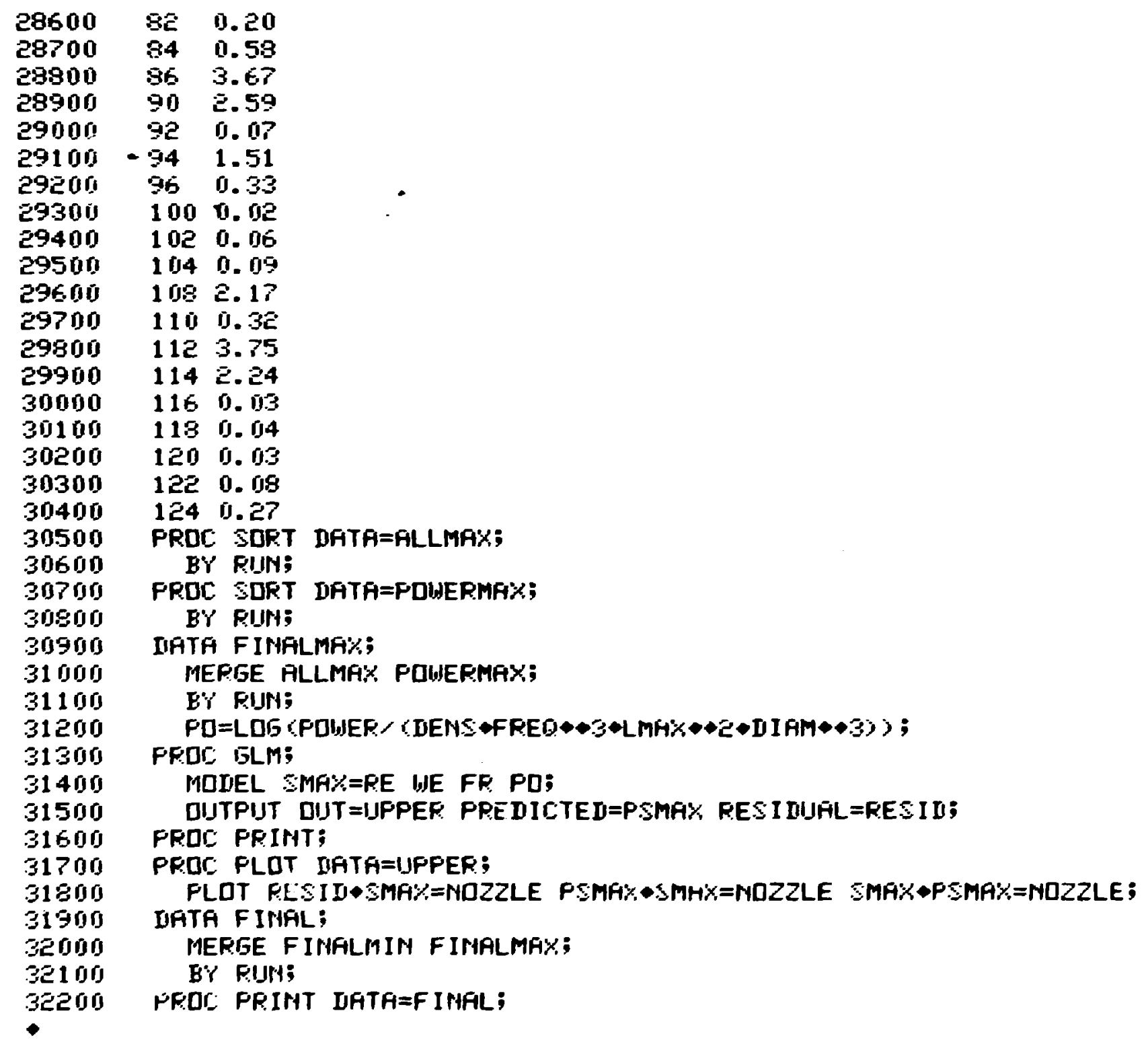




\section{5}

\subsection{Salple Cillculations}

The following somple calculations are based on the data of run 101 .

Total pressure upstrean of the nozz?: is given by:

$$
\begin{aligned}
P_{\text {total }} & =P_{\text {air }}+P_{\text {static head }} \\
& =\left[(\mathrm{ca} \mathrm{Hg})(13.6)+(\text { level })(2.54)\left(P_{\text {glycerol }} \text { water }\right)\right] \frac{98.07}{1000} \\
& =[(4.9)(13.6)+(6.5)(2.54)(1.0469)] \frac{98.07}{1000 t}
\end{aligned}
$$$$
=8.23 \mathrm{kPa}
$$

Droplet diaseter is given by (18):

$$
D=\left(\frac{6}{7} \frac{0}{f}\right)^{1 / 3}=\left(\frac{6}{\pi} \frac{0.27}{1250}\right)^{1 / 3}=744 \mathrm{ra}
$$

The natural frequency of oscillation of the droplets is calculated using Eqs. (7) through (9) of Appendix 9.1. For $20 \%$ glycol, substituting the values for all the constants into Eq. (7), this reduces to:

$$
B_{I}=66.5029\left(\frac{6}{R^{3}}\right)^{\frac{1}{2}}-\frac{0.3124}{R}\left(\frac{6}{R^{3}}\right)^{t} .
$$

and $R=0.0372 \mathrm{~cm}$ or $D=0.0744 \mathrm{~cm}$. Therefore,

$$
B_{I}=3262 \mathrm{~Hz}
$$

The velocity of the jet is given by:

$$
V=\frac{Q}{S}=\frac{Q}{\left(\frac{\pi D_{n}^{2}}{4}\right)}=\frac{4 Q}{\pi D_{n}^{2}}=\frac{(4)(0.27)}{(\pi)(0.0350)^{2}}=280.63 \mathrm{~cm} / \mathrm{s}
$$

The droplet spacing is given by: 


$$
\lambda_{\text {calculated }}=\left(\frac{v}{f}\right)(10)=\frac{280.63}{1250}(10)=2.245 \mathrm{~mm}
$$

and

where

$$
\lambda_{\text {observed }}=\frac{25.4}{{\Lambda_{d}-1}_{14-1}}=\frac{25.4}{1.954 \mathrm{~cm}}
$$

$N_{d}=$ number of Irops observed in 1-in. length of jet

\subsection{Location of Original Data}

The original data are located in ORUL Databook A-9185-6, pp. 1-56, on file at the MIT School of Chemical Engineering Practice, Bldg. 1505, ORNL. A copy of the original data, a calculation file, and a literature file are also on file.

\subsection{Nomenclature}

$A, B, C, D, E, F, F^{\prime}$

$a, b, c, d, e, g, h, k$ empirical constants

D droplet diameter, cm or um

$D_{j} \quad$ jet diameter, cm or $\mu n$

D nozzle diameter, cm or um

E voltage, volt

f frequency, $\mathrm{Hz}$

g gravitational acceleration, $\mathrm{cm} / \mathrm{s}$

I current, amp

HMTA hexame thylenetetramine

L length

M mass

$N$ numbrer of orifices

$N_{d}$ number of drops, drops/in.

$n$ principle model of oscillation, usually $n=2$ 


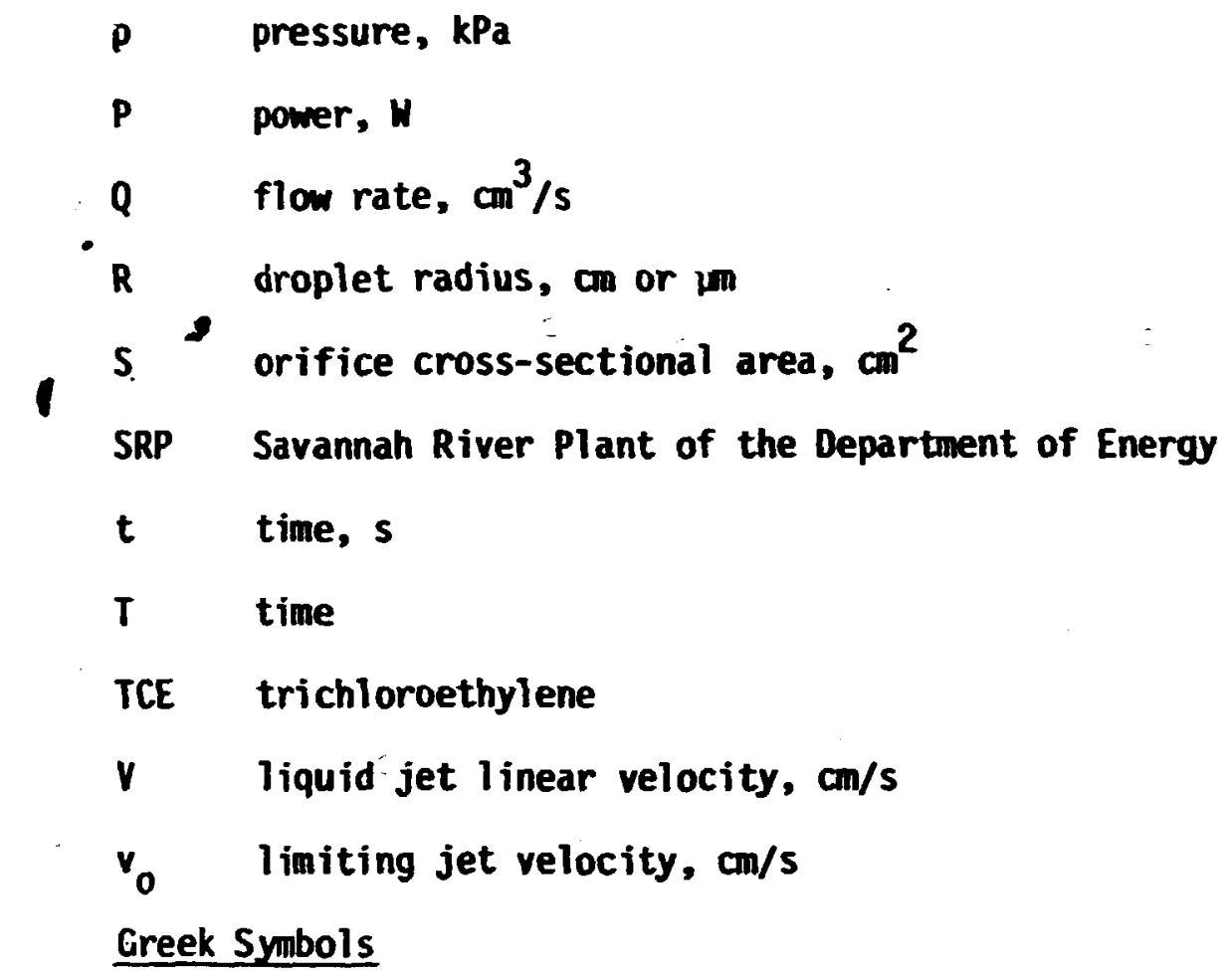

$B_{I} \quad$ natural frequency, $\mathrm{Hz}$

$\beta^{*} \quad$ frequency correction in Eq. (8), $\mathrm{Hz}$

$\Gamma$ density weighting factor in Eq. (9), g/ $\mathrm{cm}^{3}$

$\lambda_{\text {obs }}, \lambda_{\text {calc }} \begin{aligned} & \text { observed and calculated center-to-center droplet spacing, } \\ & \mathrm{cm} \text { or } \mu \mathrm{m}\end{aligned}$

p density, $\mathrm{kg} / \mathrm{m}^{3}$

$\mu \quad v i s c o s i t y$, Pa-s

$\sigma \quad$ surface tension, $N / m$

$\pi \quad 3.14159 .$.

Dimensioniess Groups

Fr iroude number $\left(V^{2} / g D_{n}\right)$

Po Puwer number $\left(P / \rho f^{3} \lambda^{2} D_{n}^{3}\right)$

Re Reynolds number $\left(D_{n} V \rho / \mu\right)$

We Weber number $\left(D_{n} v^{2} \rho / \sigma\right)$ 
$\lambda / D_{n}$ dimensionless droplet spacing

$0 / D_{n}$ diameter ratio

\section{- $\quad 9.8$ Literature References}

1. Advanced Dynamic Instruments, "Installation and Operating Instructions, 25 Watt Sol id State Oscillator/Amplifier," Serial No. 293, Dec. 18, 1980.

2. Beer, J.M., and N.A. Chigier, Combustion Aerodynamics, pp. 165-161, Hiley, New York, 1972.

3. Bond, H.D., personal communication, "Contribution to Alternative Haste Forms Progress Report," Aug. 25, 1981.

4. Cline, H.E., and T.R. Anthony, "The Effect of Harmonics on the Capillary Instability of Liquid Jets," J. Appl. Phys., $49(6), 3202$ (1978).

5. Grace, J.R., T. Wairegi, and J. Brophy, "Break-Up of Drops and Bubbles in Stagnant Media," Can. J. Chem. Engr., 56, 3 (February 1978).

6. Grant, R.P., and S. Middltman, "Newtonian Jet Stability," AIChE J., 12(4), 669 (1966).

7. Haas, P.A., "Formation of Liquid Drnps with Uniform and Controlled Diameters at Rates of $10^{3}$ and $10^{5}$ Drops Per Minute," AIChE J., 21, 383 (1975).

8. Haas, P.A., personal comunication, "Formation of Gel Particles," Aug. 18, 1981 .

9. Haas, P.A., J.M. Begovich, A.D. Ryon, and J.S. Vavruska, "Chemical Flowsheet Conditions for Preparing Urania Spheres by Internal Gelation," I\&EC Prod. Res. Dev., 19, 459 (1980).

10. Loshak, Jonathan, "Forced Oscillation of Drops in a Viscous Medium," M.S. Thesis, Univ. of Rochester, Rochester, NY, 1969.

11. Loshak, Jonathan, and C.H. Byers, "Forced Oscillation of Drops in a Viscous Medium," Chem. Eng. Sci., 28, 149 (1973).

12. Merrington, A.C., and E.G. Richardson, "The Break-Up of Liquid Jets," Proc. Phys. Soc. London, 59, 1 (1947).

13. Pimbley, H.T., and H.C. Lee, "Satellite Droplet Formation in a Liquid Jet," IBM J. Res. Dev., 21, 21 (Jan. 1977).

14. Rayleigh, Lord, "On the Instability of Jets," Proc. London Math. Soc., 10, 4 (1878). 
15. SAS Users's Gui_e, 1979 Edition, SAS Institute, Inc., Box 8000, Cary, H.C. 27511.

16. Stone, J.A., S.T. Goforth, and P.X. Saith, "Prel iniary Evaluation of Alternative Forms for Immobilization of Savannah River Plant High-Level Waste," DP-1545 (Decenber 1979).

17. Tiegs, S.M., P.A. Haas, and R.D. Spence, "The Sphere-Cal Process: Fabrication of Fuel Pellets from Gel Microspheres," ORdL/TM-6906 (Sept. 1979).

18. Williams, D.F., and C.C. Haws, "Design of and Operating Procedures for an Interim Facility for Preparing Kilogran Quantities of $\mathrm{NO}_{2}$ Spheres by Internal Gelation," ORL/TH-6950 (1980). 